\title{
Theoretically-Efficient and Practical Parallel DBSCAN
}

\author{
Yiqiu Wang \\ yiqiuw@mit.edu \\ MIT CSAIL
}

\author{
Yan $\mathrm{Gu}$ \\ ygu@ucr.edu \\ UC Riverside
}

\author{
Julian Shun \\ jshun@mit.edu \\ MIT CSAIL
}

\begin{abstract}
The DBSCAN method for spatial clustering has received significant attention due to its applicability in a variety of data analysis tasks. There are fast sequential algorithms for DBSCAN in Euclidean space that take $O(n \log n)$ work for two dimensions, sub-quadratic work for three or more dimensions, and can be computed approximately in linear work for any constant number of dimensions. However, existing parallel DBSCAN algorithms require quadratic work in the worst case. This paper bridges the gap between theory and practice of parallel DBSCAN by presenting new parallel algorithms for Euclidean exact DBSCAN and approximate DBSCAN that match the work bounds of their sequential counterparts, and are highly parallel (polylogarithmic depth). We present implementations of our algorithms along with optimizations that improve their practical performance. We perform a comprehensive experimental evaluation of our algorithms on a variety of datasets and parameter settings. Our experiments on a 36-core machine with two-way hyper-threading show that our implementations outperform existing parallel implementations by up to several orders of magnitude, and achieve speedups of up to $33 \mathrm{x}$ over the best sequential algorithms.
\end{abstract}

\section{ACM Reference Format:}

Yiqiu Wang, Yan Gu, and Julian Shun. 2020. Theoretically-Efficient and Practical Parallel DBSCAN. In Proceedings of the 2020 ACM SIGMOD International Conference on Management of Data (SIGMOD'20), June 14-19, 2020, Portland, OR, USA. ACM, New York, NY, USA, 17 pages. https://doi.org/10.1145/3318464.3380582

\section{Introduction}

Spatial clustering methods are frequently used to group together similar objects. Density-based spatial clustering of applications with noise (DBSCAN) is a popular method developed by Ester et al. [32] that is able to find good clusters of different shapes in the presence of noise without requiring

Permission to make digital or hard copies of all or part of this work for personal or classroom use is granted without fee provided that copies are not made or distributed for profit or commercial advantage and that copies bear this notice and the full citation on the first page. Copyrights for components of this work owned by others than ACM must be honored. Abstracting with credit is permitted. To copy otherwise, or republish, to post on servers or to redistribute to lists, requires prior specific permission and/or a fee. Request permissions from permissions@acm.org.

SIGMOD'20, fune 14-19, 2020, Portland, OR, USA

(c) 2020 Association for Computing Machinery.

ACM ISBN 978-1-4503-6735-6/20/06 ..\$15.00

https://doi.org/10.1145/3318464.3380582 prior knowledge of the number of clusters. The DBSCAN algorithm has been applied successfully to clustering in spatial databases, with applications in various domains such as transportation, biology, and astronomy.

The traditional DBSCAN algorithm [32] and their variants require work quadratic in the input size in the worst case, which can be prohibitive for the large data sets that need to be analyzed today. To address this computational bottleneck, there has been recent work on designing parallel algorithms for DBSCAN and its variants $[1,3,4,10,14,17,23,24,27$, $33,33,37,43,45-48,52,53,57,62,63,66-69,77,84,86-88]$. However, even though these solutions achieve scalability and speedup over sequential algorithms, in the worst-case their number of operations still scale quadratically with the input size. Therefore, a natural question is whether there exist DBSCAN algorithms that are faster both in theory and practice, and in both the sequential and parallel settings.

Given the ubiquity of datasets in Euclidean space, there has been work on faster sequential DBSCAN algorithms in this setting. Gunawan [39] and de Berg et al. [29] has shown that Euclidean DBSCAN in 2D can be solved sequentially in $O(n \log n)$ work. Gan and Tao [34] provide alternative Euclidean DBSCAN algorithms for two-dimensions that take $O(n \log n)$ work. For higher dimensions, Chen et al. [18] provide an algorithm that takes $O\left(n^{2(1-1 /(d+2))}\right.$ polylog $\left.(n)\right)$ work for $d$ dimensions, and Gan and Tao [34] improve the result with an algorithm that takes $O\left(n^{2-(2 /(\lceil d / 2\rceil+1))+\delta}\right)$ work for any constant $\delta>0$. To further reduce the work complexity, there have been approximate DBSCAN algorithms proposed. Chen et al. [18] provide an approximate DBSCAN algorithm that takes $O(n \log n)$ work for any constant number of dimensions, and Gan and Tao [34] provide a similar algorithm taking $O(n)$ expected work. However, none of the algorithms described above have been parallelized.

This paper bridges the gap between theory and practice in parallel Euclidean DBSCAN by providing new parallel algorithms for exact and approximate DBSCAN with work complexity matching that of best sequential algorithms [29, $34,39]$, and with low depth, which is the gold standard in parallel algorithm design. For exact 2D DBSCAN, we design several parallel algorithms that use either the box or the grid construction method for partitioning points [29, 39] and one of the following three methods for determining connectivity among core points: Delaunay triangulation [34], unit-spherical emptiness checking with line separation [34], 
and bichromatic closest pairs. For higher-dimensional exact DBSCAN, we provide an algorithm based on solving the higher-dimensional bichromatic closest pairs problem in parallel. Unlike many existing parallel algorithms, our exact algorithms produce the same results according to the standard definition of DBSCAN, and so we do not sacrifice clustering quality. For approximate DBSCAN, we design an algorithm that uses parallel quadtree construction and querying. Our approximate algorithm returns the same result as the sequential approximate algorithm by Gan and Tao [34].

We perform a comprehensive set of experiments on synthetic and real-world datasets using varying parameters, and compare our performance to optimized sequential implementations as well as existing parallel DBSCAN algorithms. On a 36-core machine with two-way hyper-threading, our exact DBSCAN implementations achieve $2-89 x$ (24x on average) self-relative speedup and 5-33x (16x on average) speedup over the fastest sequential implementations. Our approximate DBSCAN implementations achieve $14-44 \mathrm{x}$ (24x on average) self-relative speedup. Compared to existing parallel algorithms, which are scalable but have high overheads compared to serial implementations, our fastest exact algorithms are faster by up to orders of magnitude (16-6102x) under correctly chosen parameters. Our algorithms can process the largest dataset that has been used in the literature for exact DBSCAN, and outperform the state-of-the-art distributed RP-DBSCAN algorithm [77] by 18-577x.

The contributions of this paper are as follows.

(1) New parallel algorithms for 2D exact DBSCAN, and higherdimensional exact and approximate DBSCAN with work bounds matching that of the best existing sequential algorithms, and polylogarithmic depth.

(2) Highly-optimized implementations of our parallel DBSCAN algorithms.

(3) A comprehensive experimental evaluation showing that our algorithms achieve excellent parallel speedups over the best sequential algorithms and outperform existing parallel algorithms by up to orders of magnitude.

\section{Preliminaries}

DBSCAN Definition. The DBSCAN (density-based spatial clustering of applications with noise) problem takes as input $n$ points $\mathcal{P}=\left\{p_{0}, \ldots, p_{n-1}\right\}$, a distance function $d$, and two parameters $\epsilon$ and minPts [32]. A point $p$ is a core point if and only if $\left|\left\{p_{i} \mid p_{i} \in \mathcal{P}, d\left(p, p_{i}\right) \leq \epsilon\right\}\right| \geq$ minPts. We denote the set of core points as $C$. DBSCAN computes and outputs subsets of $\mathcal{P}$, referred to as clusters. Each point in $C$ is in exactly one cluster, and two points $p, q \in C$ are in the same cluster if and only if there exists a list of points $\bar{p}_{1}=$ $p, \bar{p}_{2}, \ldots, \bar{p}_{k-1}, \bar{p}_{k}=q$ in $C$ such that $d\left(\bar{p}_{i-1}, \bar{p}_{i}\right) \leq \epsilon$. For all non-core points $p \in \mathcal{P} \backslash C, p$ belongs to cluster $C_{i}$ if $d(p, q) \leq$ $\epsilon$ for at least one point $q \in C \cap C_{i}$. Note that a non-core point can belong to multiple clusters. A non-core point belonging to at least one cluster is called a border point and a noncore point belonging to no clusters is called a noise point. For a given set of points and parameters $\epsilon$ and minPts, the clusters returned are unique. Similar to many previous papers on parallel DBSCAN, we focus on the Euclidean distance metric in this paper. See Figure 1(a) for an illustration of the DBSCAN problem.

Gan and Tao [34] define the approximate DBSCAN problem, which in addition to the DBSCAN inputs, takes a parameter $\rho$. The definition is the same as DBSCAN, except for the connectivity rule among core points. In particular, core points within a distance of $\epsilon$ are still connected, but core points within a distance of $(\epsilon, \epsilon(1+\rho)]$ may or may not be connected. Core points with distance greater than $\epsilon(1+\rho)$ are still not connected. Due to this relaxation, multiple valid clusterings can be returned. The relaxation is what enables an asymptotically faster algorithm to be designed. A variation of this problem was described by Chen et al. [19].

Existing algorithms as well as some of our new algorithms use subroutines for solving the bichromatic closest pair $(\boldsymbol{B C P})$ problem, which takes as input two sets of points $P_{1}$ and $P_{2}$, finds the closest pair of points $p_{1}$ and $p_{2}$ such that $p_{1} \in P_{1}$ and $p_{2} \in P_{2}$, and returns the pair and their distance.

Computational Model. We use the work-depth model [25, 50] to analyze the theoretical efficiency of parallel algorithms. The work of an algorithm is the number of operations used, similar to the time complexity in the sequential RAM model. The depth is the length of the longest sequence dependence. By Brent's scheduling theorem [15], an algorithm with work $W$ and depth $D$ has overall running time $W / P+D$, where $P$ is the number of processors available. In practice, the Cilk work-stealing scheduler [9] can be used to obtain an expected running time of $W / P+O(D)$. A parallel algorithm is workefficient if its work asymptotically matches that of the best serial algorithm for the problem, which is important since in practice the $W / P$ term in the running time often dominates.

Parallel Primitives. We give an overview of the primitives used in our new parallel algorithms, and show their work and depth bounds in Table 1. We use implementations of these primitives from the Problem Based Benchmark Suite (PBBS) [76], an open-source library.

Prefix sum takes as input an array $A$ of length $n$, and returns the array $\left(0, A[0], A[0]+A[1], \ldots, \sum_{i=0}^{n-2} A[i]\right)$ as well as the overall sum, $\sum_{i=0}^{n-1} A[i]$. Prefix sum can be implemented by first adding the odd-indexed elements to the even-indexed elements in parallel, recursively computing the prefix sum for the even-indexed elements, and finally using the results on the even-indexed elements to update the odd-indexed elements in parallel. This algorithm takes $O(n)$ work and $O(\log n)$ depth [50]. 

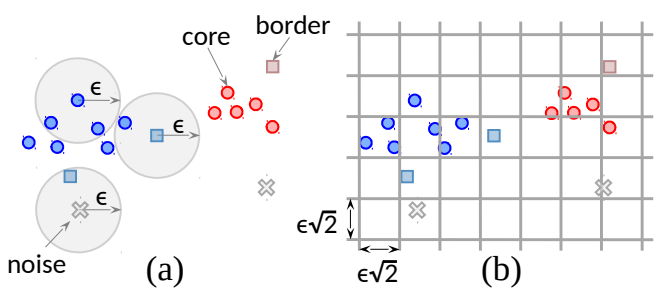

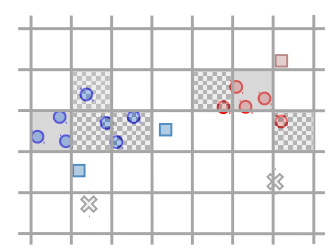

(c)

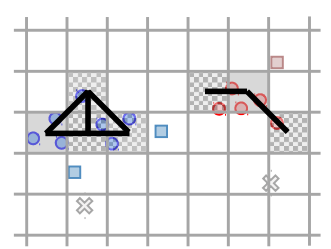

(d)

Figure 1: An example of DBSCAN and basic concepts in two dimensions. Here we set minPts $=3$ and $\epsilon$ as drawn. In (a), the points are categorized into core points (circles) in two clusters (red and blue), border points (squares) that belong to the clusters, and noise points (crosses). Using the grid method for cell construction, the algorithm constructs cells with side length $\epsilon / \sqrt{2}$ (diagonal length $\epsilon$ ), as shown in (b). The cells with at least minPts points are marked as core cells (solid gray cells in (c)), while points in other cells try to check if they have minPts points within a distance of $\epsilon$. If so, the associated cells are marked as core cells as well (checkered cells in (c)). To construct the cell graph, we create an edge between two core cells if the closest pair of points from the two cells is within a distance of $\epsilon$ (shown in (d)). Each connected component in the cell graph is a unique cluster. Border points are assigned to clusters that they are within $\epsilon$ distance from.

\begin{tabular}{llll}
\hline & Work & Depth & Reference \\
\hline Prefix sum, Filter & $O(n)$ & $O(\log n)$ & {$[50]$} \\
Comparison sort & $O(n \log n)$ & $O(\log n)$ & {$[21,50]$} \\
Integer sort & $O(n)$ & $O(\log n)$ & {$[80]$} \\
Semisort & $O(n)^{\dagger}$ & $O(\log n)^{*}$ & {$[38]$} \\
Merge & $O(n)$ & $O(\log n)$ & {$[50]$} \\
Hash table & $O(n)^{*}$ & $O(\log n)^{*}$ & {$[36]$} \\
2D Delaunay triangulation & $O(n \log n)^{*}$ & $O(\log n)^{*}$ & {$[71]$} \\
\hline
\end{tabular}

Table 1: Work and depth bounds for parallel primitives. ${ }^{\dagger}$ indicates an expected bound and * indicates a high-probability bound. The integer sort is for a polylogarithmic key range. The cost of the hash table is for $n$ insertions or queries.

Filter takes an array $A$ of size $n$ and a predicate $f$, and returns a new array $A^{\prime}$ containing elements $A[i]$ for which $f(A[i])$ is true, in the same order as in $A$. We first construct an array $P$ of size $n$ with $P[i]=1$ if $f(A[i])$ is true and $P[i]=0$ otherwise. Then we compute the prefix sum of $P$. Finally, for each element $A[i]$ where $f(A[i])$ is true, we write it to the output array $A^{\prime}$ at index $P[i]$ (i.e., $A^{\prime}[P[i]]=A[i]$ ). This algorithm also takes $O(n)$ work and $O(\log n)$ depth [50].

Comparison sorting sorts $n$ elements based on a comparison function. Parallel comparison sorting can be done in $O(n \log n)$ work and $O(\log n)$ depth [21,50]. We use a cacheefficient samplesort [8] from PBBS which samples $\sqrt{n}$ pivots on each level of recursion, partitions the keys based on the pivots, and recurses on each partition in parallel.

We also use integer sorting, which sorts integer keys from a polylogarithmic range in $O(n)$ work and $O(\log n)$ depth [80]. The algorithm partitions the keys into sub-arrays and in parallel across all partitions, builds a histogram on each partition serially. It then uses a prefix sum on the counts of each key per partition to determine unique offsets into a global array for each partition. Finally, all partitions write their keys into unique positions in the global array in parallel.

Semisort takes as input $n$ key-value pairs, and groups pairs with the same key together, but with no guarantee on the relative ordering among pairs with different keys. Semisort also returns the number of distinct groups. We use the implementation from [38], which is available in PBBS.
The algorithm first hashes the keys, and then selects a sample of the keys to predict the frequency of each key. Based on the frequency of keys in the sample, we classify them into "heavy keys" and "light keys", and assign appropriately-sized arrays for each heavy key and each range of light keys. Finally, we insert all keys into random locations in the appropriate array and sort within the array. This algorithm takes $O(n)$ expected work and $O(\log n)$ depth with high probability. ${ }^{1}$

Merge takes two sorted arrays, $A$ and $B$, and merges them into a single sorted array. If the sum of the lengths of the inputs is $n$, this can be done in $O(n)$ work and $O(\log n)$ depth [50]. The algorithm takes equally spaced pivots from $A$ and does a binary search for each pivot in $B$. Each sub-array between pivots in $A$ has a corresponding sub-array between the binary search results in $B$. Then it repeats the above process for each pair, except that equally spaced pivots are taken from the sub-array from $B$ and binary searches are done in the sub-array from $A$. This creates small subproblems each of which can be solved using a serial merge, and the results are written to a unique range of indices in the final output. All subproblems can be processed in parallel.

For parallel hash tables, we can perform $n$ insertions or queries taking $O(n)$ work and $O(\log n)$ depth w.h.p. [36]. We use the non-deterministic concurrent linear probing hash table from [75], which uses an atomic update to insert an element to an empty location in its probe sequence, and continues probing if the update fails.

The Delaunay triangulation on a set of points in 2D contains triangles among every triple of points $p_{1}, p_{2}$, and $p_{3}$ such that there are no other points inside the circumcircle defined by $p_{1}, p_{2}$, and $p_{3}$ [28]. Delaunay triangulation can be computed in parallel in $O(n \log n)$ work and $O(\log n)$ depth w.h.p. [71]. We use the randomized incremental algorithm from PBBS, which inserts points in parallel into the triangulation in rounds, such that the updates to the triangulation in each round by different points do not conflict [7].

\footnotetext{
${ }^{1}$ We say that a bound holds with high probability (w.h.p.) on an input of size $n$ if it holds with probability at least $1-1 / n^{c}$ for a constant $c>0$.
} 


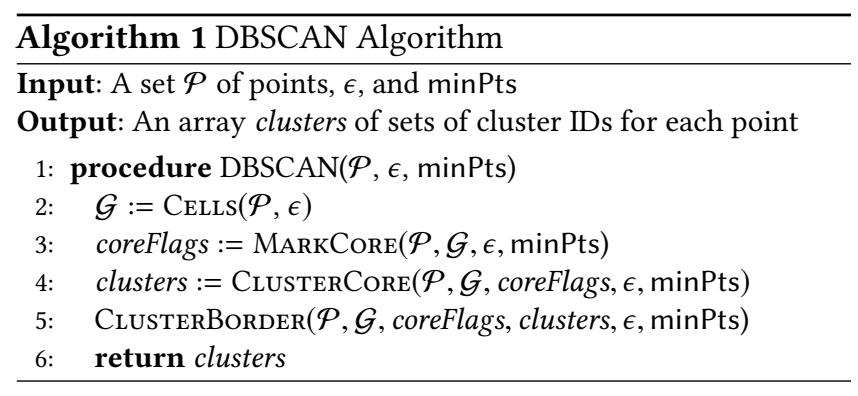

\section{DBSCAN Algorithm Overview}

This section reviews the high-level structure of existing sequential DBSCAN algorithms [29, 34, 39] as well as our new parallel algorithms. The high-level structure is shown in Algorithm 1, and an illustration of the key concepts are shown in Figure 1(b)-(d).

We place the points into disjoint $d$-dimensional cells with side-length $\epsilon / \sqrt{d}$ based on their coordinates (Line 2 and Figure 1(b)). The cells have the property that all points inside a cell are within a distance of $\epsilon$ from each other. Then on Line 3 and Figure 1(c), we mark the core points. On Line 4 , we generate the clusters for core points as follows. We create a graph containing one vertex per core cell (a cell containing at least one core point), and connect two vertices if the closest pair of core points from the two cells is within a distance of $\epsilon$. We refer to this graph as the cell graph. This step is illustrated in Figure 1(d). We then find the connected components of the cell graph to assign cluster IDs to points in core cells. On Line 5, we assign cluster IDs for border points. Finally, we return the cluster labels on Line 6 .

All of our algorithms share this common structure. In Section 4, we introduce our 2D algorithms, and in Section 5, we introduce our algorithms for higher dimensions. We analyze the complexity of our algorithms in Section 6.

\section{2D DBSCAN Algorithms}

This section presents our parallel algorithms for implementing each line of Algorithm 1 in two dimensions. The cells can be constructed either using a grid-based method or a box-based method, which we describe in Sections 4.1 and 4.2, respectively. Section 4.3 presents our algorithm for marking core points. We present several methods for constructing the cell graph in Section 4.4. Finally, Section 4.5 describes our algorithm for clustering border points.

\subsection{Grid Computation}

In the grid-based method, the points are placed into disjoint cells with side-length $\epsilon / \sqrt{2}$ based on their coordinates, as done in the sequential algorithms by Gunawan [39] and de Berg et al. [29]. A hash table is used to store only the nonempty cells, and a serial algorithm simply inserts each point into the cell corresponding to its coordinates.

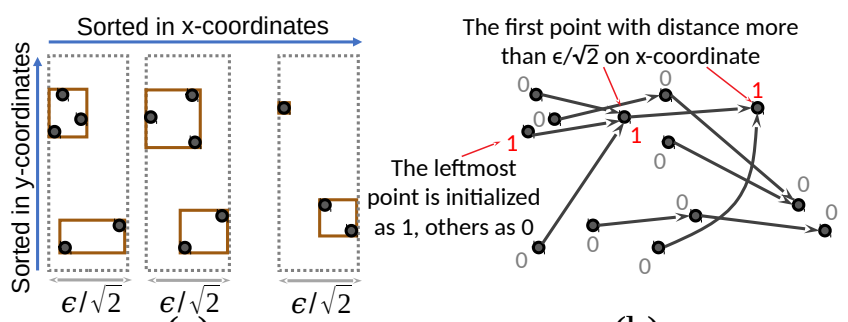

(a)

(b)

Figure 2: Parallel box method construction. In (a), the gray dashed rectangles correspond to strips and the brown solid rectangles correspond to box cells. To compute the strips, we create a pointer from each point to the first point with an $x$-coordinate that is more than $\epsilon / \sqrt{2}$ larger. We initialize the leftmost point with a value of 1 and all other points with a value of 0 . As shown in (b), after running pointer jumping, the points at the beginning of strips have values of 1 and all other points have values of 0 . We apply the same procedure in each strip on the $y$-coordinates to obtain the boxes.

Parallelization. The challenge in parallelization is in distributing the points to the cells in parallel while maintaining work-efficiency. While a comparison sort could be used to sort points by their cell IDs, this approach requires $O(n \log n)$ work and is not work-efficient. We observe that semisort (see Section 2) can be used to solve this problem work-efficiently. The key insight here is that we only need to group together points in the same cell, and do not care about the relative ordering of points within a cell or between different cells. We apply a semisort on an array of length $n$ of key-value pairs, where each key is the cell ID of a point and the value is the ID of the point. This also returns the number of distinct groups (non-empty cells).

We then create a parallel hash table of size equal to the number of non-empty cells, where each entry stores the bounding box of a cell as the key, and the number of points in the cell and a pointer to the start of its points in the semisorted array as the value. We can determine neighboring cells of a cell $g$ with arithmetic computation based on $g$ 's bounding box, and then look up each neighboring cell in the hash table, which returns the information for that cell if it is non-empty.

\subsection{Box Computation}

In the box-based method, we place the points into disjoint 2dimensional bounding boxes with side-length at most $\epsilon / \sqrt{d}$, which are the cells.

Existing sequential solutions [29,39] first sort all points by $x$-coordinate, then scan through the points, grouping them into strips of width $\epsilon / \sqrt{2}$ and starting a new strip when a scanned point is further than distance $\epsilon / \sqrt{2}$ from the beginning of the strip. It then repeats this process per strip in the $y$-dimension to create cells of side-length at most $\epsilon / \sqrt{2}$. This step is shown in Figure 2(a). Pointers to neighboring cells are stored per cell. This is computed for all cells in each $x$-dimensional strip $s$ by merging $s$ with each of strips $s-2$, 
$s-1, s+1$, and $s+2$, as these are the only strips that can contain cells with points within distance $\epsilon$. For each merge, we compare the bounding boxes of the cells in increasing $y$-coordinate, linking any two cells that may possibly have points within $\epsilon$ distance.

Parallelization. We now describe the method for assigning points to strips, which is illustrated in Figure 2(b). Let $p_{x}$ be the $x$-coordinate of point $p$. We create a linked list where each point is a node. The node for point $p$ stores a pointer to the node for point $q$ (we call $q$ the parent of $p$ ), where $q$ is the point with the smallest $x$-coordinate such that $p_{x}+\epsilon / \sqrt{2}<$ $q_{x}$. Each point can determine its parent in $O(\log n)$ work and depth by binary searching the sorted list of points.

We then assign a value of 1 to the node with the smallest $x$-coordinate, and 0 to all other nodes. We run a pointer jumping routine on the linked list where on each round, every node passes its value to its parent and updates its pointer to point to the parent of its parent [50]. The procedure terminates when no more pointers change in a round. In the end, every node with a value of 1 will correspond to the point at the beginning of a strip, and all nodes with a value of 0 will belong to the strip for the closest node to the left with a value of 1 . This gives the same strips as the sequential algorithm, since all nodes marked 1 will correspond to the closest point farther than $\epsilon / \sqrt{2}$ from the point of the previously marked node. For merging to determine cells within distance $\epsilon$, we use the parallel merging algorithm described in Section 2.

\subsection{Mark Core}

Illustrated in Figure 1(c), the high-level idea in marking the core points is as follows: first, if a cell contains at least minPts points then all points in the cell are core points, as it is guaranteed that all the points inside a cell will be within $\epsilon$ to any other point in the same cell; otherwise, each point $p$ computes the number of points within its $\epsilon$-radius by checking its distance to points in all neighboring cells (defined as cells that could possibly contain points within a distance of $\epsilon$ to the current cell), and marking $p$ as a core point if the number of such points is at least minPts. For a constant dimension, only a constant number of neighboring cells need to be checked.

Parallelization. Our parallel algorithm for marking core points is shown in Algorithm 2. We create an array coreFlags of length $n$ that marks which points are core points. The array is initialized to all 0's (Line 2). We then loop through all cells in parallel (Line 3 ). If a cell contains at least minPts points, we mark all points in the cell as core points in parallel (Line 4-6). Otherwise, we loop through all points $p$ in the cell in parallel, and for each neighboring cell $h$ we count the number of points within a distance of $\epsilon$ to $p$, obtained using a RANGECount $(p, \epsilon, h)$ query (Lines $8-11)$ that reports the number of points in $h$ that are no more than $\epsilon$ distance from

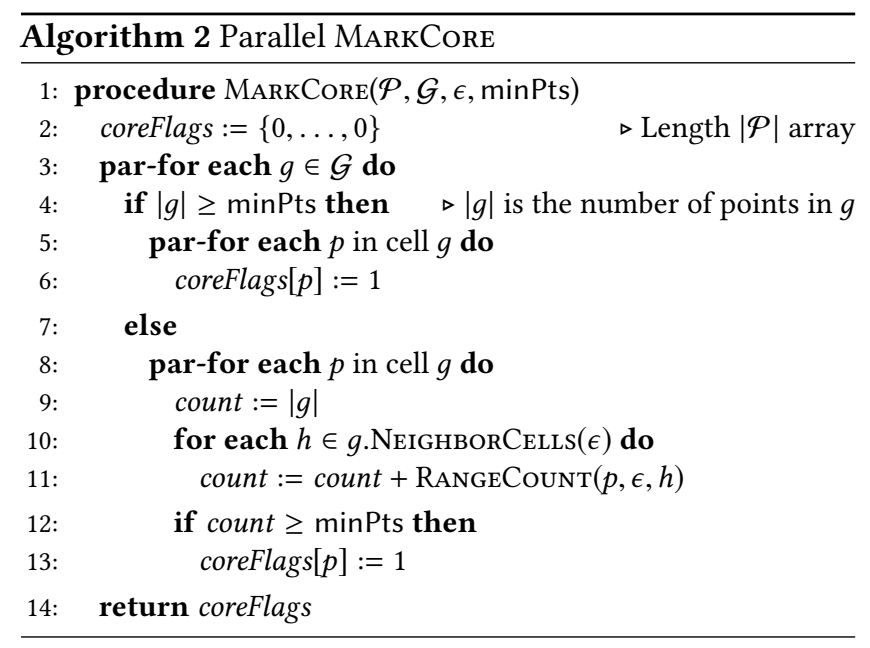

$p$. The RangeCount $(p, \epsilon, h)$ query can be implemented by comparing $p$ to all points in each neighboring cell $h$ in parallel, followed by a parallel prefix sum to obtain the number of points in the $\epsilon$-radius. If the total count is at least minPts, then $p$ is marked as a core point (Lines 12-13).

\subsection{Cluster Core}

We present three approaches for determining the connectivity between cells in the cell graph. After obtaining the cell graph, we run a parallel connected components algorithm to cluster the core points. For the BCP-based approach, we describe an optimization that merges the BCP computation with the connected components computation using a lock-free union-find data structure.

BCP-based Cell Graph. The problem of determining cell connectivity can be solved by computing the BCP of core points between two cells (recall the definition in Section 2), and checking whether the distance is at most $\epsilon$.

Each cell runs a BCP computation with each of its neighboring cells to check if they should be connected in the cell graph. We execute all BCP calls in parallel, and furthermore each BCP call can be implemented naively in parallel by computing all pairwise distances in parallel, writing them into an array containing point pairs and their distances, and applying a prefix sum on the array to obtain the BCP. We apply two optimizations to speed up individual BCP calls: (1) we first filter out points further than $\epsilon$ from the other cell beforehand as done by Gan and Tao [34], and (2) we iterate only until finding a pair of points with distance at most $\epsilon$, at which point we abort the rest of the BCP computation, and connect the two cells. Filtering points can be done using a parallel filter. To parallelize the early termination optimization, it is not efficient to simply parallelize across all the point comparisons as this will lead to a significant amount of wasted work. Instead, we divide the points in each cell into fixed-sized blocks, and iterate over all pairs of blocks. For each pair of blocks, we compute the distances of all pairs 

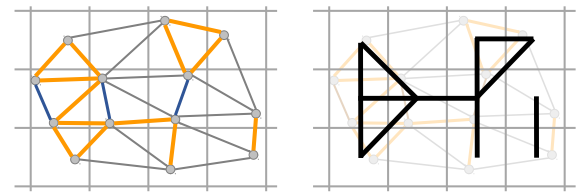

Figure 3: Using Delaunay triangulation (DT) to construct the cell graph in 2D. (Left) We construct the DT for all core points, and an edge in the DT can either be inside a cell (dark blue), or across cells with length no more than $\epsilon$ (orange), or with length more than $\epsilon$ (gray). (Right) An orange edge will add the associated edge in the cell graph, and in this example, there are two clusters.

of points between the two blocks in parallel by writing their distances into an array. We then take the minimum distance in the array using a prefix sum, and return if the minimum is at most $\epsilon$. This approach reduces the wasted work over the naive parallelization, while still providing ample parallelism within each pair of blocks.

Triangulation-based Cell Graph. In two dimensions, Gunawan [39] describes a special approach using Voronoi diagrams. In particular, we can efficiently determine whether a core cell should be connected to a neighboring cell by finding the nearest core point from the neighboring cell to each of the core cell's core points. Gan and Tao [34] and de Berg et al. [29] show that a Delaunay triangulation can also be used to determine connectivity in the cell graph. In particular, if there is an edge in the Delaunay triangulation between two core cells with distance at most $\epsilon$, then those two cells are connected. This process is illustrated in Figure 3. The proof of correctness is described in [29,34].

To compute Delaunay triangulation or Voronoi diagram in parallel, Reif and Sen present a parallel algorithm for constructing Voronoi diagrams and Delaunay triangulations in two dimensions. We use the parallel Delaunay triangulation implementation from PBBS [7, 76], as described in Section 2.

Unit-spherical emptiness checking-based (USEC) Cell Graph. Gan and Tao [34] (who attribute the idea to Bose et al. [12]) describe an algorithm for solving the unit-spherical emptiness checking (USEC) with line separation problem to determine cell connectivity. The high-level idea is to compute the wavefront of each cell containing the area within distance $\epsilon$ to any point in this cell, and check if core points in neighboring cells are contained in this region. We design a new parallel algorithm for this approach and evaluate it in Section 7. Due to space constraints, we present details in the full version of the paper [83].

Reducing Cell Connectivity Queries. We now present an optimization that merges the cell graph construction with the connected components computation using a parallel lockfree union-find data structure to maintain the connected components on-the-fly. This technique is used in both the

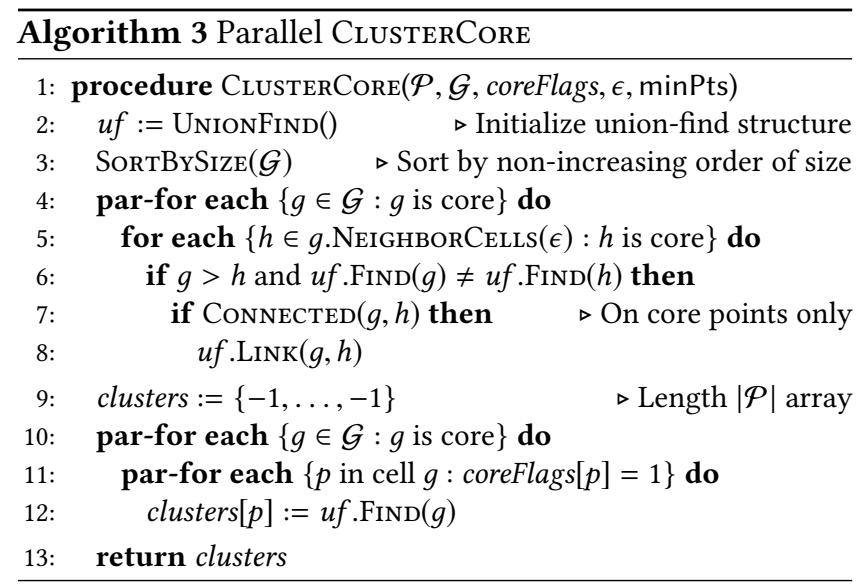

BCP approach and USEC approach for cell graph construction. The pseudocode is shown in Algorithm 3. The idea is to only run a cell connectivity query between two cells if they are not yet in the same component (Line 6), which can reduce the total number of connectivity queries. For example, assume that cells $a, b$, and $c$ belong to the same component. After connecting $a$ with $b$ and $b$ with $c$, we can avoid the connectivity check between $a$ and $c$ by checking their respective components in the union-find structure beforehand. This optimization was used by Gan and Tao [34] in the sequential setting, and we extend it to the parallel setting. We also only check connectivity between two cells at most once by having the cell with higher ID responsible for checking connectivity with the cell with a lower ID (Line 6).

When constructing the cell graph and checking connectivity, we use a heuristic to prioritize the cells based on the number of core points in the cells, and start from the cells with more points, as shown on Line 3 . This is because cells with more points are more likely to have higher connectivity, hence connecting the nearby cells together and pruning their connectivity queries. This optimization can be less efficient in parallel, since a connectivity query could be executed before the corresponding query that would have pruned it in the sequential execution. To overcome this, we group the cells into batches, and process each batch in parallel before moving to the next batch. We refer to this new approach as bucketing, and show experimental results for it in Section 7.

\subsection{Cluster Border}

To assign cluster IDs for border points. We check all points not yet assigned a cluster ID, and for each point $p$, we check all of its neighboring cells and add it to the clusters of all neighboring cells with a core point within distance $\epsilon$ to $p$.

Parallelization. Our algorithm is shown in Algorithm 4. We loop through all cells with fewer than minPts points in parallel, and for each such cell we loop over all of its noncore points $p$ in parallel (Lines $2-3$ ). On Lines $4-7$, we check all core points in the current cell $g$ and all neighboring cells, 


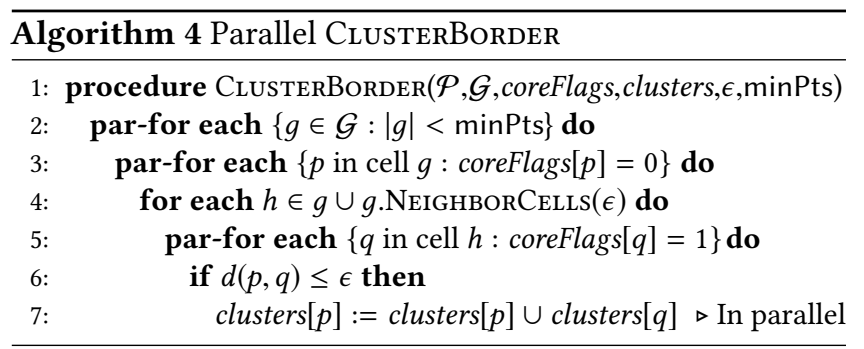

and if any are within distance $\epsilon$ to $p$, we add their clusters to $p$ 's set of clusters (recall that border points can belong to multiple clusters).

\section{Higher-dimensional Exact and Approximate DBSCAN}

The efficient exact and approximate algorithms for higherdimensional DBSCAN are also based on the high-level structure of Algorithm 1, and are extensions of some of the techniques for two-dimensional DBSCAN described in Section 4. They use the grid-based method for assigning points to cells (Section 4.1). Algorithms 2, 3, and 4 are used for marking core points, clustering core points, and clustering border points, respectively. However, we use two major optimizations on top of the 2D algorithms: a $k$-d tree for finding neighboring cells and a quadtree for answering range counting queries.

\subsection{Finding Neighboring Cells}

The number of possible neighboring cells grows exponentially with the dimension $d$, and so enumerating all possible neighboring cells can be inefficient in practice for higher dimensions (although still constant work in theory). Therefore, instead of implementing NEIGHBORCELLs by enumerating all possible neighboring cells, we first insert all cells into a $k$-d tree [6], which enables us to perform range queries to obtain just the non-empty neighboring cells. The construction of our $k$-d tree is done recursively, and all recursive calls for children nodes are executed in parallel. We also sort the points at each level in parallel and pass them to the appropriate child. Queries do not modify the $k$-d tree, and can all be performed in parallel. Since a cell needs to find its neighboring cells multiple times throughout the algorithm, we cache the result on its first query to avoid repeated computation.

\subsection{Range Counting}

While RANGECOUNT queries can be implemented theoreticallyefficiently in DBSCAN by checking all points in the target cell, there is a large overhead for doing so in practice. In higher-dimensional DBSCAN, we construct a quadtree data structure for each cell to answer RANGECOUNT queries. The structure of a quadtree is illustrated in Figure 4. A cell of side-length $\epsilon / \sqrt{d}$ is recursively divided into $2^{d}$ sub-cells of the same size until the sub-cell becomes empty. This forms a tree where each sub-cell is a node and its children are the up to $2^{d}$ non-empty sub-cells that it divides into. Each node of

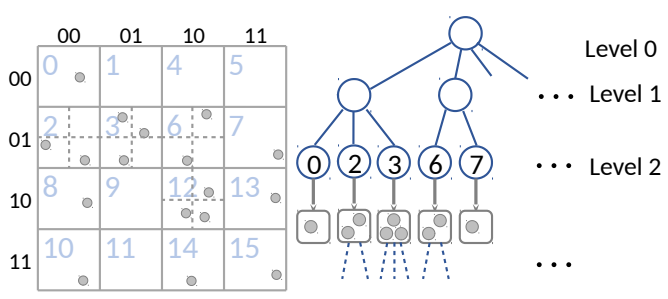

Figure 4: A cell (left) and its corresponding quadtree data structure (right).

the tree stores the number of points contained in its corresponding sub-cell. Queries do not modify the quadtrees and are therefore all executed in parallel. We now describe how to construct the quadtrees in parallel.

Parallel Quadtree Construction. The construction procedure recursively divides each cell into sub-cells. Each node of the tree has access to the points contained in its sub-cell in a contiguous subarray that is part of a global array (e.g., by storing a pointer to the start of its points in the global array as well as the number of points that it represents). We use an integer sort on keys from the range $\left[0, \ldots, 2^{d}-1\right]$ to sort the points in the subarray based on which of the $2^{d}$ sub-cells it belongs to. Now the points belonging to each of the child nodes are contiguous, and we can recursively construct the up to $2^{d}$ non-empty child nodes independently in parallel by passing in the appropriate subarray.

To reduce construction time, we set a threshold for the number of points in a sub-cell, below which the node becomes a leaf node. This reduces the height of the tree but makes leaf nodes larger. In addition, we avoid unnecessary tree node traversal by ensuring that each tree node has at least two non-empty children: when processing a cell, we repeatedly divide the points until they fall into at least two different sub-cells.

Range Counting in MarkCore. RangeCount queries are used in marking core points in Algorithm 2. For each cell, a quadtree containing all of its points is constructed in parallel. Then the RANGECount $(p, \epsilon, h)$ query reports the number of points in cell $h$ that are no more than $\epsilon$ distance from point $p$. Instead of naively looping through all points in $h$, we initiate a traversal of the quadtree starting from cell $h$, and recursively search all children whose sub-cell intersects with the $\epsilon$-radius of $p$. When reaching a leaf node on a query, we explicitly count the number of points contained in the $\epsilon$-radius of the query point.

Exact DBSCAN. For higher-dimensional exact DBSCAN, one of our implementations uses RANGECOUNT queries when computing BCPs in Algorithm 3. For each core cell, we build a quadtree on its core points in parallel. Then for each core point $p$ in each core cell $g$, we issue a RANGECOUNT query to each of its neighboring core cells $h$ and connect $g$ and 
$h$ in the cell graph if the range query returns a non-zero count of core points. Since we do not need to know the actual count, but only whether or not it is non-zero, our range query is optimized to terminate once such a result can be determined. We combine this with the optimization of reducing cell connectivity queries described in Section 4.4

Approximate DBSCAN. For approximate DBSCAN, the sequential algorithm of Gan and Tao [34] follows the high-level structure of Algorithm 1 using the grid-based cell structure. The only difference is in the cell graph construction, which is done using approximate RANGECount queries.

In the quadtree for approximate RANGECOUNT, each cell of side-length $\epsilon / \sqrt{d}$ is still recursively divided into $2^{d}$ subcells of the same size, but until either the sub-cell becomes empty or has side-length at most $\epsilon \rho / \sqrt{d}$. The tree has maximum depth $l=1+\left\lceil\log _{2} 1 / \rho\right\rceil$. We use a modified version of our parallel quadtree construction method to parallelize approximate DBSCAN.

An approximate RAngeCount $(p, \epsilon, h, \rho)$ query takes as input a point $p$, and returns an integer that is between the number of points in the $\epsilon$-radius and the number of points in the $\epsilon(1+\rho)$-radius of $p$ that are in $h$, (when using approximate RANGECOUNT, all relevant methods takes an additional parameter $\rho$ ). If the answer is non-zero, then the core cell containing $p$ is connected to core cell $h$. Our query implementation starts a traversal of the quadtree from $h$, and recursively searches all children whose sub-cell intersects with the $\epsilon$-radius of $p$. As done in exact DBSCAN, our query is optimized to terminate once a zero count or a non-zero count can be determined. Once either a leaf node is reached or a node's sub-cell is completely contained in the $\epsilon(1+\rho)$ radius of $p$, the search on that path terminates. Queries do not modify the quadtree and can all be executed in parallel.

\section{Analysis}

This section analyzes the theoretical complexity of our algorithms, showing that they are work-efficient and have polylogarithmic depth.

\subsection{D Algorithms}

Grid Computation. In our parallel algorithm presented in Section 4.1, creating $n$ key-value pairs can be done in $O(n)$ work and $O(1)$ depth in a data-parallel fashion. Semisorting takes $O(n)$ expected work and $O(\log n)$ depth w.h.p. Constructing the hash table and inserting non-empty cells into it takes $O(n)$ work and $O(\log n)$ depth w.h.p. The overall cost of the parallel grid computation is therefore $O(n)$ work in expectation and $O(\log n)$ depth w.h.p.

Box Computation. The serial algorithm [29, 39] uses $O(n \log n)$ work, including sorting, scanning the points to assign them to strips and cells, and merging strips. However, the span is $O(n)$ since in the worst case there can be $O(n)$ strips.
Parallel comparison sorting takes $O(n \log n)$ work and $O(\log n)$ depth. Therefore, sorting the points by $x$-coordinate, and each strip by $y$-coordinate can be done in $O(n \log n)$ work and $O(\log n)$ depth overall. Parent finding using binary search for all points takes $O(n \log n)$ work and $O(1)$ depth. For pointer jumping, the longest path in the linked list halves on each round, and so the algorithm terminates after $O(\log n)$ rounds. We do $O(n)$ work per round, leading to an overall work of $O(n \log n)$. The depth is $O(1)$ per round, for a total of $O(\log n)$ overall. We repeat this process for the points in each strip, but in the $y$-direction, and the work and depth bounds are the same. For assigning pointers to neighboring cells for each cell, we use a parallel merging algorithm, which takes $O(n)$ work and $O(\log n)$ depth. The pointers are stored in an array, accessible in constant work and depth.

MARKCore. For cells with at least minPts points, we spend $O(n)$ work overall marking their points as core points (Lines 4-6 of Algorithm 2). All cells are processed in parallel, and all points can be marked in parallel, giving $O(1)$ depth.

For all cells with fewer than minPts points, each point only needs to execute a range count query on a constant number of neighboring cells $[34,39]$. RANGECount $(p, \epsilon, h)$ compares $p$ to all points in neighboring cell $h$ in parallel. Across all queries, each cell will only be checked by $O$ (minPts) many points, and so the overall work for range counting is $O(n$. minPts). Therefore, Lines 8-13 of Algorithm 2 takes $O(n$. minPts) work. All points are processed in parallel, and there are a constant number of RANGECOUNT calls per point, each of which takes $O(\log n)$ depth for a parallel prefix sum to obtain the number of points in the $\epsilon$-radius. Therefore, the depth for range counting is $O(\log n)$.

The work for looking up the neighbor cells is $O(n)$ and depth is $O(\log n)$ w.h.p. using the parallel hash table that stores the non-empty cells. Therefore, parallel MARKCORE takes $O(n \cdot \operatorname{minPts})$ work and $O(\log n)$ depth w.h.p.

Cell Graph Construction. Reif and Sen present a parallel algorithm for constructing Voronoi diagrams and Delaunay triangulations in two dimensions in $O(n \log n)$ work and $O(\log n)$ depth w.h.p. [71]. For the Voronoi diagram approach, each nearest neighbor query can be answered in $O(\log n)$ work, which is used to check whether two cells should be connected and can be applied in parallel. Each cell will only execute a constant number of queries, and so the overall complexity is $O(n \log n)$ work and $O(\log n)$ depth w.h.p. For the Delaunay triangulation approach, we can simply apply a parallel filter over all of the edges in the triangulation, keeping the edges between different cells with distance at most $\epsilon$. The cost of the filter is dominated by the cost of constructing the Delaunay triangulation. In the full version of the paper [83], we show that the USEC-based approach takes $O(n \log n)$ work and $O\left(\log ^{3} n\right)$ depth. 
Connected Components. After the cell graph that contains $O(n)$ points and edges are constructed, we run connected components on the cell graph. This step can be done in parallel in $O(n)$ work and $O(\log n)$ depth w.h.p. using parallel connectivity algorithms [22, 35, 41, 42, 70].

ClusterBorder. Using a similar analysis as done for marking core points, it can be shown that assigning cluster IDs to border points takes $O(n \cdot \operatorname{minPts})$ work sequentially $[29,39]$. In parallel, since there are a constant number of neighboring cells for each non-core point, and all points in neighboring cells as well as all non-core points are checked in parallel, the depth is $O(1)$ for the distance comparisons. Looking up the neighboring cells can be done in $O(n)$ work and $O(\log n)$ depth w.h.p. using our parallel hash table. Adding cluster IDs to border point's set of clusters, while removing duplicates at the same time, can be done using parallel hashing in linear work and $O(\log n)$ depth w.h.p. The work is $O(n \cdot \operatorname{minPts})$ since we do not introduce any asymptotic work overhead compared to the sequential algorithm.

Overall, we have the following theorem.

THEOREM 6.1. For a constant value of minPts, 2D Euclidean DBSCAN can be computed in $O(n \log n)$ work and $O(\log n)$ depth w.h.p.

\subsection{Higher-dimensional Algorithm}

The theoretically-efficient algorithms for grid construction, marking core points, connected components, and clustering border points extend naturally from the $2 \mathrm{D}$ algorithms with linear work and $O(\log n)$ depth w.h.p. In the full version of the paper [83], we analyze higher-dimensional cell graph construction using a more sophiscated higher-dimensional $\mathrm{BCP}$ algorithm and show that it takes sub-quadratic work and polylogarithmic depth. This gives the following theorem.

Theorem 6.2. For a constant value of minPts, Euclidean DBSCAN can be solved in $O\left((n \log n)^{4 / 3}\right)$ expected work for $d=$ 3 and $O\left(n^{2-(2 /([d / 2\rceil+1))+\delta}\right)$ expected work for any constant $\delta>$ 0 for $d>3$, and polylogarithmic depth with high probability.

The theoretically-efficient $\mathrm{BCP}$ algorithm for higher dimensions is not practical, and so our implementation of DBSCAN uses the approach described in Section 4.4 in higher dimensions, which takes quadratic work.

\subsection{Approximate Algorithm}

The algorithms for grid construction, marking core points, connected components, and clustering border points are the same as the exact algorithms, and so we only analyze approximate cell graph construction in the approximate algorithm based on the quadtree introduced in Section 5.2. The quadtree has $l=1+\left\lceil\log _{2} 1 / \rho\right\rceil$ levels and can be constructed in $O\left(n^{\prime} l\right)$ work sequentially for a cell with $n^{\prime}$ points. A hash table is used to map non-empty cells to their quadtrees, which takes $O(n)$ work w.h.p. to construct. Using a fact from [5], Gan and Tao show that the number of nodes visited by a query is $O\left(1+(1 / \rho)^{d-1}\right)$. Therefore, for constant $\rho$ and $d$, all of the quadtrees can be constructed in a total of $O(n)$ work w.h.p., and queries can be answered in $O(1)$ expected work.

All of the quadtrees can be constructed in parallel. To parallelize the construction of a quadtree for a cell with $n^{\prime}$ points, we sort the points on each level in $O\left(n^{\prime}\right)$ work and $O\left(\log n^{\prime}\right)$ depth using parallel integer sorting [80], since the keys are integers in a constant range. In total, this gives $O\left(n^{\prime} l\right)$ work and $O\left(l \log n^{\prime}\right)$ depth per quadtree. We use a parallel hash table to map non-empty cells to their quadtrees, which takes $O(n)$ work and $O(\log n)$ depth w.h.p. to construct. To construct the cell graph, all core points issue a constant number of queries to neighboring cells in parallel. The $O(n)$ hash table queries can be done in $O(n)$ work and $O(\log n)$ depth w.h.p. and thus cell graph construction has the same complexity. This gives the following theorem.

THEOREM 6.3. For constant values of minPts and $\rho$, our approximate Euclidean DBSCAN algorithm takes $O(n)$ work and $O(\log n)$ depth with high probability.

\section{Experiments}

This section presents experiments comparing our exact and approximate algorithms as well as existing algorithms.

Datasets. We use the synthetic seed spreader (SS) datasets produced by Gan and Tao's generator [34]. The generator produces points generated by a random walk in a local neighborhood, but jumping to a random location with some probability. SS-simden and $\boldsymbol{S S}$-varden refer to the datasets with similar-density and variable-density clusters, respectively. We also use a synthetic dataset called UniformFill that contains points distributed uniformly at random inside a bounding hypergrid with side length $\sqrt{n}$, where $n$ is the total number of points. The points have double-precision floating point values, but we scaled them to integers when testing Gan and Tao's implementation, which requires integer coordinates. We generated the synthetic datasets with 10 million points (unless specified otherwise) for dimensions $d=2,3,5,7$.

In addition, we use the following real-world datasets, which contain points with double-precision floating point values.

(1) Household [30] is a 7-dimensional dataset with 2, 049, 280 points excluding the date-time information.

(2) GeoLife [89] is a 3-dimensional dataset with 24, 876, 978 points. This dataset contains user location data (longitude, latitude, altitude), and its distribution is extremely skewed.

(3) Cosmo50 [59] is a 3-dimensional dataset with 321, 065, 547 points. We extracted the $x, y$, and $z$ coordinate information to construct the 3-dimensional dataset.

(4) OpenStreetMap [40] is a 2-dimensional dataset with 2,770, 238, 904 points, containing GPS location data. 
(5) TeraClickLog [26] is a 13-dimensional dataset with 4,373, 472, 329 points containing feature values and click feedback of online advertisements. As far as we know, TeraClickLog is the largest dataset used in the literature for exact DBSCAN.

We performed a search on $\epsilon$ and minPts for the synthetic datasets and chose the default parameters to be those that output a correct clustering. For the SS datasets, the default parameters that we use are similar to those found by Gan and Tao [34]. For ease of comparison, the default parameters for Household are the same as Gan and Tao [34] and the default parameters for GeoLife, Cosmo50, OpenStreetMap, and TeraClickLog are same as RP-DBSCAN [77]. For approximate DBSCAN, we set $\rho=0.01$, unless specified otherwise.

Testing Environment. We perform all of our experiments on Amazon EC2 machines. We use a c5.18xlarge machine for testing of all datasets other than Cosmo50, OpenStreetMap, and TeraClickLog. The c5.18xlarge machine has $2 \times$ Intel Xeon Platinum $8124 \mathrm{M}(3.00 \mathrm{GHz})$ CPUs for a total for a total of 36 two-way hyper-threaded cores, and 144 GB of RAM. We use a r5.24xlarge machine for the three larger datasets just mentioned. The r5.24xlarge machine has $2 \times$ Intel Xeon Platinum $8175 \mathrm{M}(2.50 \mathrm{GHz})$ CPUs for a total of 48 two-way hyper-threaded cores, and 768 GB of RAM. By default, we use all of the cores with hyper-threading on each machine. We compile our programs with the g++ compiler (version 7.4) with the -03 flag, and use Cilk Plus for parallelism [60].

\subsection{Algorithms Tested}

We implement the different methods for marking core points and BCP computation in exact and approximate DBSCAN for $d \geq 3$, and present results for the fastest versions, which are described below.

- our-exact: This exact implementation implements the RANGECOUNT query in marking core points by scanning through all points in the neighboring cell in parallel described in Section 4.3. For determining connectivity in the cell graph, it uses the BCP method described in Section 4.4.

- our-exact-qt: This exact implementation implements the RANGECOUnT query supported by the quadtree described in Section 5.2. For determining connectivity in the cell graph, it uses the BCP method described in Section 4.4.

- our-approx: This approximate implementation implements the RANGECount query in marking core points by scanning through all points in the neighboring cell in parallel, and uses the quadtree for approximate RANGECOUNT queries in cell graph construction described in Section 5.2.

- our-approx-qt: This approximate implementation is the same as our-approx except that it uses the RANGECounT query supported by the quadtree described in Section 5.2 for marking core points.
We append the -bucketing suffix to the names of these implementations when using the bucketing optimization described in Section 4.4 .

For $d=2$, we have six implementations that differ in whether they use the grid or the box method to construct cells and whether they use BCP, Delaunay triangulation, or USEC with line separation to construct the cell graph. We refer to these as our-2d-grid-bcp, our-2d-grid-usec, our-2d-griddelaunay, our-2d-box-bcp, our-2d-box-usec, and our-2dbox-delaunay.

We note that our exact algorithms return the same answer as the standard DBSCAN definition, and our approximate algorithms return answers that satisfy Gan and Tao's approximate DBSCAN definition (see Section 2).

We compare with the following implementations:

- Gan\&Tao-v2 [34] is the state-of-the-art serial implementation for both exact and approximate DBSCAN. Gan\&Tao$v 2$ only accepts integer values between 0 and 100,000 , and so when running their code we scaled the datasets up into this integer range and scaled up the $\epsilon$ value accordingly to achieve a consistent clustering output with other methods.

- pdsdbscan [68] is the implementation of the parallel disjointset exact DBSCAN by Patwary et al. compiled with OpenMP.

- hpdbscan [37] is the implementation of parallel exact DBSCAN by Gotz et al. compiled with OpenMP. We modified the source code to remove the file output code.

- rpdbscan [77] is the state-of-the-art distributed implementation for DBSCAN using Apache Spark. We note that their variant does not return the same result as DBSCAN. We tested rpdbscan on the same machine that we used, and also report the timings in [77], which were obtained using at least as many cores as our largest machine.

\subsection{Experiments for $d \geq 3$}

We first evaluate the performance of the different algorithms for $d \geq 3$. In the following plots, data points that did not finish within an hour are not shown.

Influence of $\epsilon$ on Parallel Running Time. In this experiment, we fix the default value of minPts corresponding to the correct clustering, and vary $\epsilon$ within a range centered around the default $\epsilon$ value. Figure 5 shows the parallel running time vs. $\epsilon$ for the different implementations. In general, both pdsdbscan and hpdbscan becomes slower with increasing $\epsilon$. This is because they use pointwise range queries, which get more expensive with larger $\epsilon$. Our methods tend to improve with increasing $\epsilon$ because there are fewer cells leading to a smaller cell graph, which speeds up computations on the graph. Our implementations significantly outperform pdsdbscan and hpdbscan on all of the data points.

We observe a spike in plot Figure 5(f) when $\epsilon=608$. The implementations that mark core points by scanning through all points in neighboring cells spend a significant amount 


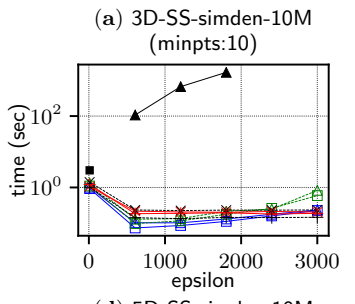

(d) 5D-SS-simden-10M (minpts:100)

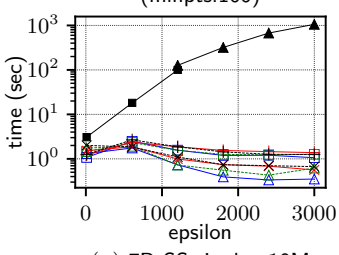

(g) 7D-SS-simden-10M (minpts:10)

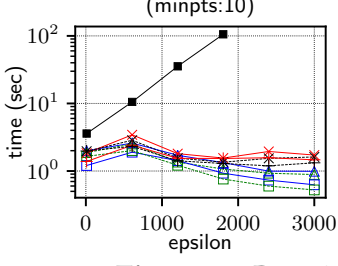

(b) 3D-SS-varden-10M (minpts:100)

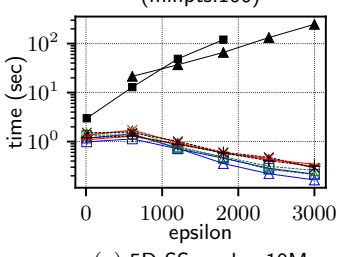

(e) 5D-SS-varden-10M (minpts:10)

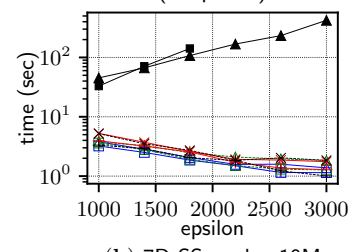

(h) 7D-SS-varden-10M (minpts:10)

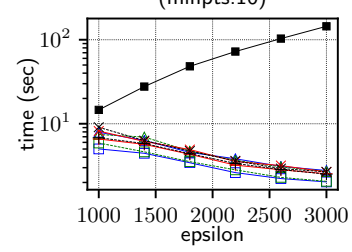

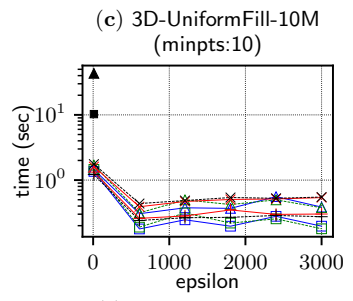

(f) 5D-UniformFill-10M (minpts:100)

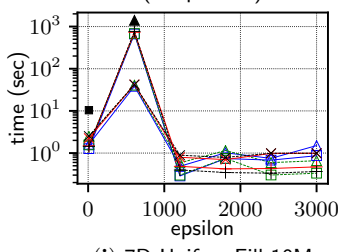

(i) 7D-UniformFill-10M (minpts:10)

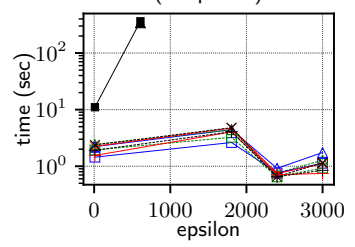

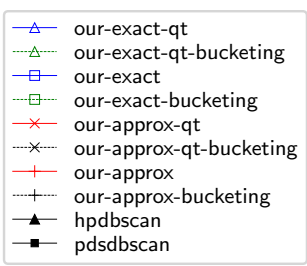

(j) 3D-GeoLife-24.9M (minpts:100)

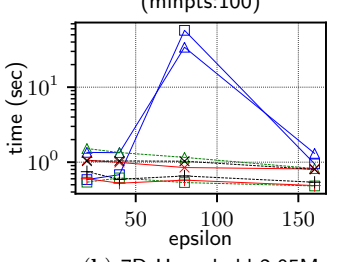

(k) 7D-Household-2.05M (minpts:100)

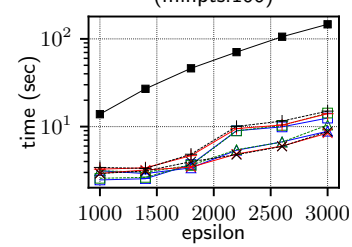

of time in that phase; in comparison, the quadtree versions perform better because of their more optimized range counting. There is also a spike in Figure 5(j) when $\epsilon=80$. Our exact implementation spends a significant amount of time in cell graph construction. This is because the GeoLife dataset is heavily skewed, certain cells could contain significantly more points. When many cell connectivity queries involve these cells, the quadratic nature using the BCP approach in our-exact makes the cost of queries expensive. On the contrary, methods using the quadtree for cell graph construction (our-exact-qt, our-approx-qt, and our-approx) tend to have consistent performance across the $\epsilon$ values. For the spike in Figure 5(j), it is interesting to see that the bucketing implementations, our-exact-qt-bucketing and our-exact-bucketing, are significantly faster than our-exact-qt and our-exact because many of the expensive connectivity queries are pruned.

Influence of minPts on Parallel Running Time. In this experiment, we fix the default value of $\epsilon$ for a dataset and vary minPts over a range from 10 to 10,000. Figure 6 shows that our implementations have an increasing trend in running time as minPts increases in most cases. This is consistent with our analysis in Section 6.1 that the overall work for marking core points is $O(n \cdot \operatorname{minPts})$. In contrast, minPts does not have much impact on the performance of hpdbscan and pdsdbscan because their range queries, which dominate the total running times, do not depend on minPts. Our implementations outperform hpdbscan and pdsdbscan for almost all values of minPts. Figures 6(d) and 6(g) suggests that $h p d b$ scan can surpass our performance for certain datasets when
$\operatorname{minPts}=10,000$. However, as suggested by Schubert et al. [74], the minPts value used in practice is usually much smaller, and based on our observation, a minPts value of at most 100 usually gives the correct clusters.

Parallel Speedup. To the best of our knowledge, Gan\&Tao$v 2$ is the fastest existing serial implementation both for exact and approximate DBSCAN. However, we find that across all of our datasets, our serial implementations are faster than theirs by an average of $5.18 \mathrm{x}$ and $1.52 \mathrm{x}$ for exact DBSCAN and approximate DBSCAN, respectively. In Figure 7, we compare the speedup of the parallel implementations under different thread counts over the best serial baselines for each dataset and choice of parameters. We also show the self-relative speedups for one dataset in Figure 8 and note that the trends are similar on other datasets. For these experiments, we use parameters that generate the correct clusters. Our implementations obtain very good speedups on most datasets, achieving speedups of 5-33x (16x on average) over the best serial baselines. Additionally, the self-relative speedups of our exact and approximate methods are 2-89x (24x on average) and $14-44 x$ (24x on average), respectively. Although $h p d b s c a n$ and $p d s d b s c a n$ achieve good self-relative speedup (22-31x and 7-20x, respectively), they fail to outperform the serial implementation on most of the datasets. Compared to $h p d b s c a n$ and $p d s d b s c a n$, we are faster by up to orders of magnitude (16-6102x).

Our speedup on the GeoLife dataset (Figure 7(j)) is low due to the high skewness of cell connectivity queries caused by the skewed point distribution, however the parallel running 


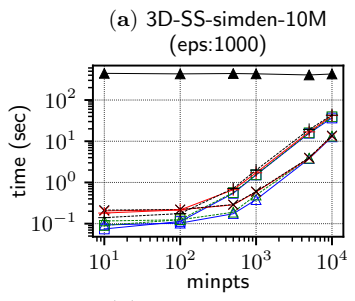

(d) 5D-SS-simden-10M

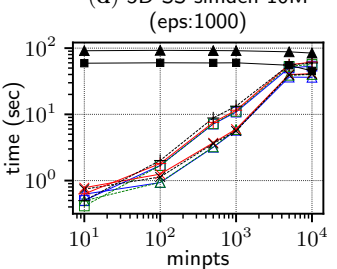

(g) 7D-SS-simden-10M (eps:2000)

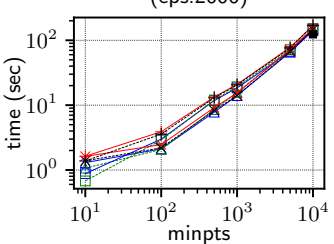

Figure 6: Running

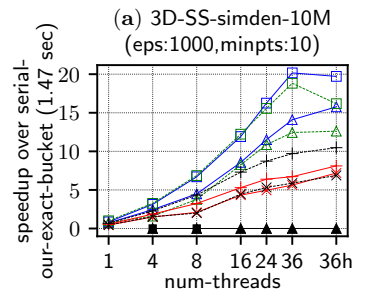

ত্ষ্口 (d) 5D-SS-simden-10M

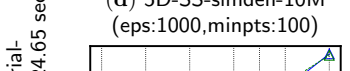

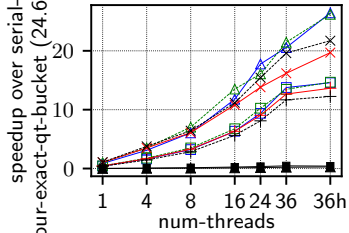

今 num-threads 1 s

I্ $\quad$ (g) 7D-SS-simden-10M

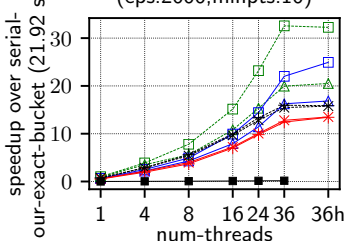

(b) 3D-SS-varden-10M

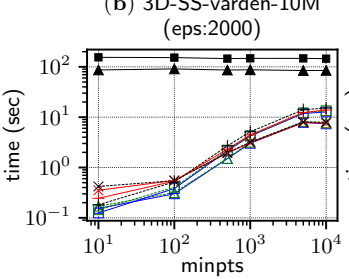

(e) 5D-SS-varden-10M (eps:3000)

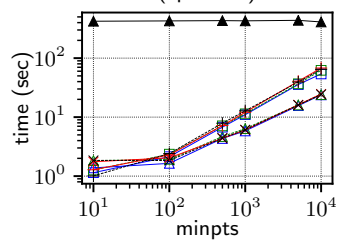

(h) 7D-SS-varden-10M (eps:3000)

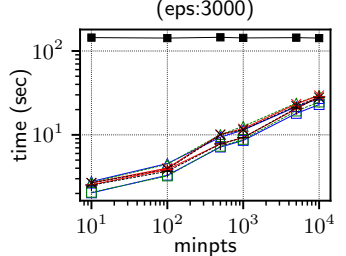

me vs. minPts on 36 cores

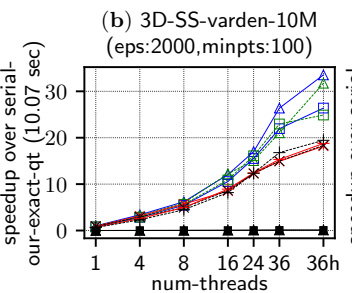

(e) 5D-SS-varden-10M
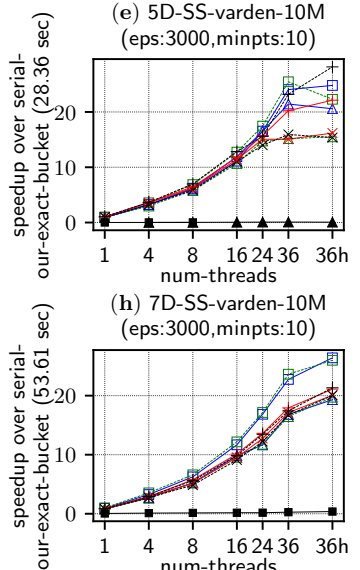

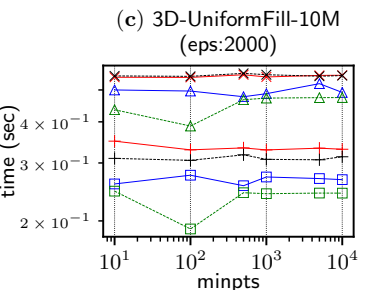

(f) 5D-UniformFill-10M (eps:2000)

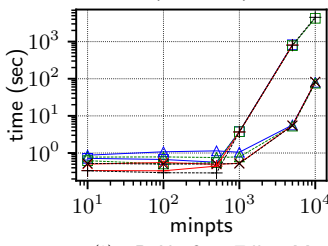

(i) 7D-UniformFill-10M

(eps:2000)

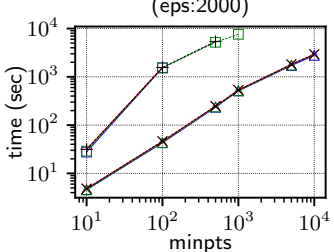

ith hyper-threading. The $y$ -

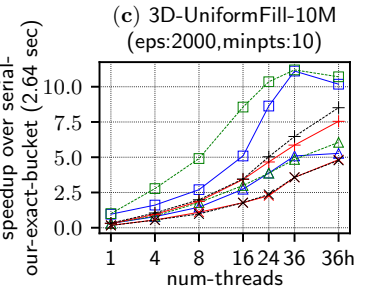

(f) 5D-UniformFill-10M

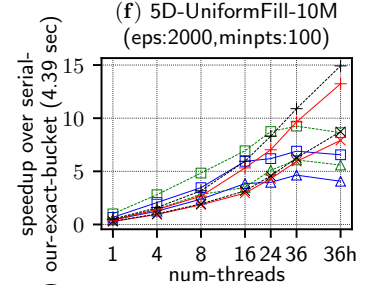

(i) 7D-UniformFill-10M

(eps:2000, minpts:10)

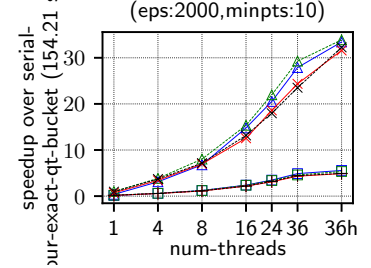

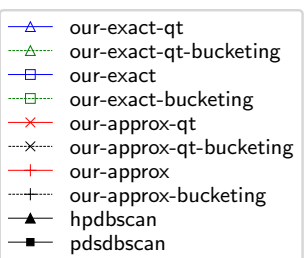

(j) 3D-GeoLife-24.9M (eps:40)

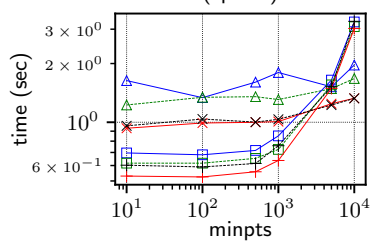

(k) 7D-Household-2.05M (eps:2000)

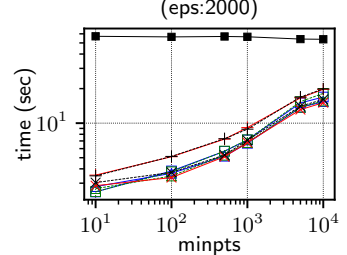

axes are in log-scale.
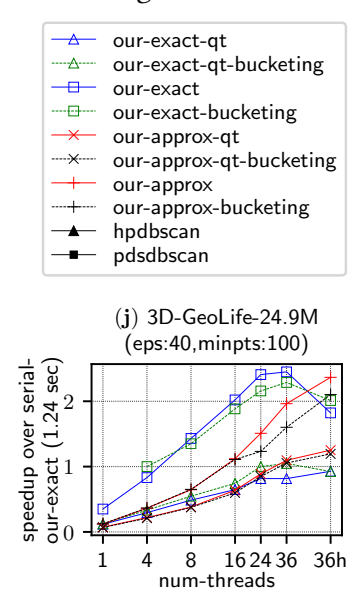

(k) 7D-Household-2.05M

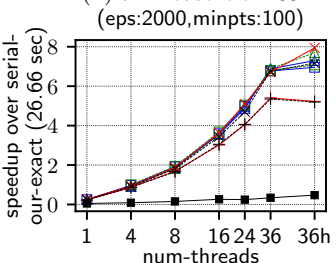

Figure 7: Speedup of implementations over the best serial baselines vs. thread count. The best serial baseline and its running time for each dataset is shown on the $y$-axis label. " $36 \mathrm{~h}$ " on the $x$-axes refers to 36 cores with hyper-threading.

time is reasonable (less than 1 second). In contrast, $h p d b s c a n$ and pdsdbscan did not terminate within an hour.

The bucketing heuristic achieved the best parallel performance for several of the datasets (Figures 5(f), (g), and (j); Figures 6(c) and (j); and Figures 7(c), (f), (g), and (j)). In general, the bucketing heuristic greatly reduces the number of connectivity queries during cell graph construction, but in some cases it can reduce parallelism and/or increase overhead due to sorting. We also observe a similar trend on all methods where bucketing is applied.

We also implemented our own parallel baseline based on the original DBSCAN algorithm [32]. We use a parallel $k$-d tree, and all points perform queries in parallel to find all neighbors in their $\epsilon$-radius to check if they should be a core 


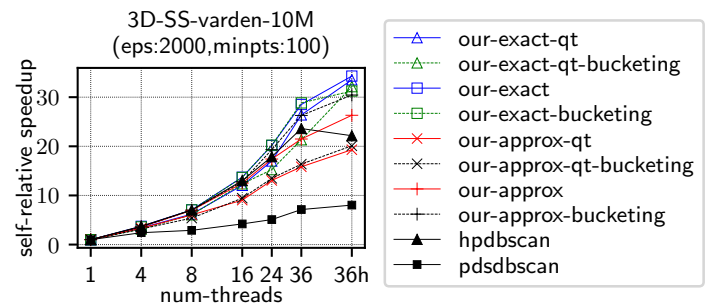

Figure 8: Self-relative speedup of implementations vs. thread count. " $36 \mathrm{~h}$ " on the $x$-axis refers to 36 cores with hyper-threading.
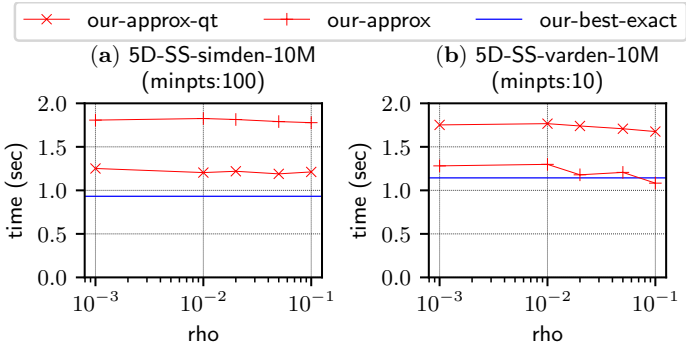

Figure 9: Running time vs. $\rho$ on 36 cores with hyper-threading.

point. However, the baseline was over 10x slower than our fastest parallel implementation for datasets with the correct parameters, and hence we do not show it in the plots.

Influence of $\rho$ on Parallel Running Time. Figure 9 shows the effect of varying $\rho$ for our two approximate DBSCAN implementations. We also show our best exact method as a baseline. We only show plots for two datasets as the trend was similar in other datasets. We observe a small decrease in running time as $\rho$ increases, but find that the approximate methods are still mostly slower than the best exact method. On average, for the parameters corresponding to correct clustering, we find that our best exact method is $1.24 \mathrm{x}$ and 1.53x faster than our best approximate method when running in parallel and serially, respectively; this can also be seen in Figure 7. Schubert et al. [74] also found exact DBSCAN to be faster than approximate DBSCAN for appropriately-chosen parameters, which is consistent with our observation.

Large-scale Datasets. In Table 2, we show the running times of our-exact on large-scale datasets. We compare with the reported numbers for the state-of-the-art distributed implementation rpdbscan, which use 48 cores distributed across 12 machines [77], as well as numbers for rpdbscan on our machines. The purpose of this experiment is to show that we are able to efficiently process large datasets using just a multicore machine. GeoLife was run on the 36 core machine whereas others were run on the 48 core machine due to their larger memory footprint. We see that our-exact achieves a 18-577x speedup over rpdbscan using the same or a fewer number of cores. We believe that this speedup is due to lower communication costs in shared-memory as well as a better algorithm. Even though TeraClickLog is significantly larger than the other datasets, our running times are not proportionally larger. This is because for the parameters chosen by [77], all points fall into one cell. Therefore, in our implementation all points are core points and are trivially placed into the only cluster. In contrast, rpdbscan incurs communication costs in partitioning the points across machines and merging the clusters from different machines together.

\subsection{Experiments for $d=2$}

In Figure 10, we show the performance of our six 2D algorithms as well as hpdbscan and pdsdbscan on the synthetic datasets. We show the running time while varying $\epsilon$, minPts, number of points, or number of threads. We first note that all of our implementations are significantly faster than hpdbscan and pdsdbscan. In general, we found the grid-based implementations to be faster than the box-based implementations due to the higher cell construction time of the boxed-based implementations. We also found the Delaunay triangulationbased implementations to be significantly slower than the BCP and USEC-based methods due to the high overhead of computing the Delaunay triangulation. The fastest implementation overall was our-2d-grid-bcp.

\section{Related Work}

$\mathrm{Xu}$ et al. [87] provide the first parallel exact DBSCAN algorithm, called PDBSCAN, based on a distributed $R^{*}$-tree. Arlia and Coppola [4] present a parallel DBSCAN implementation that replicates a sequential $R^{*}$-tree across machines to process points in parallel. Coppola and Vanneschi [23] design a parallel algorithm using a queue to store core points, where each core point is processed one at a time but their neighbors are checked in parallel to see whether they should be placed at the end of the queue. Januzaj et al. [52, 53] design an approximate DBSCAN algorithm based on determining representative points on different local processors, and then running a sequential DBSCAN on the representatives. Brecheisen et al. [14] parallelize a version of DBSCAN optimized for complex distance functions [13].

Patwary et al. [67] present PDSDBSCAN, a multicore and distributed algorithm for DBSCAN using a union-find data structure for connecting points. Their union-find data structure is lock-based whereas ours is lock-free. Patwary et al. $[66,69]$ also present distributed DBSCAN algorithms that are approximate but more scalable than PDSDBSCAN. Hu et al. [46] design PS-DBSCAN, an implementation of DBSCAN using a parameter server framework. Gotz et al. [37] present HPDBSCAN, an algorithm for both shared-memory and distributed-memory based on partitioning the data among processors, running DBSCAN locally on each partition, and then merging the clusters together. Very recently, Sarma et al. [73] present a distributed algorithm, $\mu$ DBSCAN, and report a running time of 41 minutes for clustering one billion 3-dimensional points using a cluster of 32 nodes. Our running times on the larger 13-dimensional TeraClickLog dataset are significantly faster (under 30 seconds on 48 cores). 


\begin{tabular}{|c|c|c|c|c|c|c|c|c|c|c|c|c|c|c|c|c|}
\hline & \multicolumn{4}{|c|}{ GeoLife } & \multicolumn{4}{|c|}{ Cosmo50 } & \multicolumn{4}{|c|}{ OpenStreetMap } & \multicolumn{4}{|c|}{ TeraClickLog } \\
\hline$\epsilon$ & 20 & 40 & 80 & 160 & 0.01 & 0.02 & 0.04 & 0.08 & 0.01 & 0.02 & 0.04 & 0.08 & 1500 & 3000 & 6000 & 12000 \\
\hline our-exact & 0.541 & 0.617 & 0.535 & 0.482 & 41.8 & 5.51 & 4.69 & 3.03 & 41.4 & 43.2 & 40 & 44.5 & 26.8 & 26.9 & 27.0 & 27.6 \\
\hline rpdbscan (our machine) & 29.13 & 27.92 & 32.04 & 27.81 & 3750 & 562.0 & 576.9 & 672.6 & - & - & - & - & - & - & $\begin{array}{lll}- & \\
\end{array}$ & - \\
\hline rpdbscan ([77]) & 36 & 33 & 28 & 27 & 960 & 504 & 438 & 432 & 3000 & 1720 & 1200 & 840 & 15480 & 7200 & 3540 & 1680 \\
\hline
\end{tabular}

Table 2: Parallel running times (seconds) for our-exact and rpdbscan. The value of minPts is set to 100. GeoLife was run on the 36 core machine and the other datasets were run on the 48 core machine. For rpdbscan, we omit timings for experiments that encountered exceptions or did not complete within 1 hour. We also include the distributed running times reported in [77] that used as many cores as our machines.

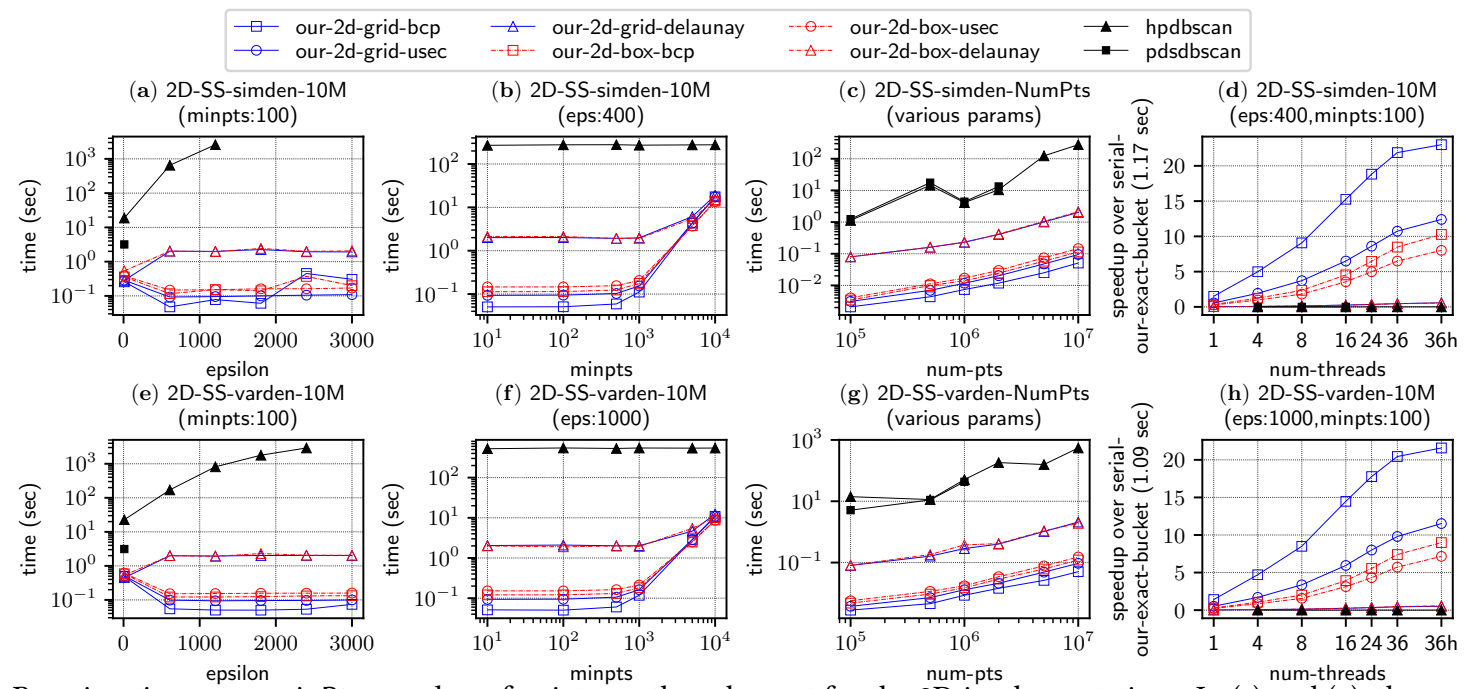

Figure 10: Running time vs. $\epsilon$, minPts, number of points, or thread count for the $2 \mathrm{D}$ implementations. In (c) and (g), the parameters are chosen for each input size such that the algorithm outputs the correct clustering. In (d) and (h), " $36 \mathrm{~h}$ " on the $x$-axis refers to 36 cores with hyper-threading. The $y$-axes in (a)-(c) and (e)-(g) are in log-scale.

Exact and approximate distributed DBSCAN algorithms have been designed using MapReduce [3, 27, 33, 45, 47, 57, $86,88]$ and Spark [24, 43, 48, 62, 63, 77]. RP-DBSCAN [77], an approximate DBSCAN algorithm, has been shown to be the state-of-the-art for MapReduce and Spark. GPU implementations of DBSCAN have also been designed $[1,10,17,84]$.

In addition to parallel solutions, there have been optimizations proposed to speed up sequential DBSCAN $[13,58,64]$. DBSCAN has also been generalized to other definitions of neighborhoods [72]. Furthermore, there have been variants of DBSCAN proposed in the literature, which do not return the same result as the standard DBSCAN. IDBSCAN [11], FDBSCAN [61], GF-DBSCAN [78], I-DBSCAN [82], GNDBSCAN [49], Rough-DBSCAN [81], and DBSCAN++ [51] use sampling to reduce the number of range queries needed. ElSonbaty et al. [31] presents a variation that partitions the dataset, runs DBSCAN within each partition, and merges together dense regions. GriDBSCAN [65] uses a similar idea with an improved scheme for partitioning and merging. Other partitioning based algorithms include PACA-DBSCAN [54], APSCAN [20], and AA-DBSCAN [56]. DBSCAN* and HDBSCAN* are variants of DBSCAN where only core points are included in clusters [16]. Other variants use approximate neighbor queries to speed up DBSCAN $[44,85]$.
OPTICS [2], SUBCLU [55], and GRIDBSCAN [79], are hierarchical versions of DBSCAN that compute DBSCAN clusters on different parameters, enabling clusters of different densities to more easily be found. POPTICS [68] is a parallel version of OPTICS based on concurrent union-find.

\section{Conclusion}

We have presented new parallel algorithms for exact and approximate Euclidean DBSCAN that are both theoreticallyefficient and practical. Our algorithms are work-efficient and have polylogarithmic depth, making them highly parallel. Our experiments demonstrate that our solutions achieve excellent parallel speedup and significantly outperform existing parallel DBSCAN solutions. Future work includes designing theoretically-efficient and practical parallel algorithms for variants of DBSCAN and hierarchical versions of DBSCAN.

Acknowledgements. This research was supported by DOE Early Career Award \#DE-SC0018947, NSF CAREER Award \#CCF-1845763, Google Faculty Research Award, DARPA SDH Award \#HR0011-18-3-0007, and Applications Driving Architectures (ADA) Research Center, a JUMP Center co-sponsored by SRC and DARPA. 


\section{References}

[1] Guilherme Andrade, Gabriel Ramos, Daniel Madeira, Rafael Sachetto, Renato Ferreira, and Leonardo Rocha. 2013. G-DBSCAN: A GPU Accelerated Algorithm for Density-based Clustering. Procedia Computer Science 18 (2013), 369 - 378.

[2] Mihael Ankerst, Markus M. Breunig, Hans-Peter Kriegel, and Jörg Sander. 1999. OPTICS: Ordering Points to Identify the Clustering Structure. In ACM International Conference on Management of Data (SIGMOD). 49-60.

[3] Antonio Cavalcante Araujo Neto, Ticiana Linhares Coelho da Silva, Victor Aguiar Evangelista de Farias, José Antonio F. Macêdo, and Javam de Castro Machado. 2015. G2P: A Partitioning Approach for Processing DBSCAN with MapReduce. In Web and Wireless Geographical Information Systems. 191-202.

[4] Domenica Arlia and Massimo Coppola. 2001. Experiments in Parallel Clustering with DBSCAN. In European Conference on Parallel Processing (Euro-Par). 326-331.

[5] Sunil Arya and David M. Mount. 2000. Approximate range searching. Computational Geometry 17, 3 (2000), 135 - 152.

[6] Jon Louis Bentley. 1975. Multidimensional Binary Search Trees Used for Associative Searching. Commun. ACM 18, 9 (Sept. 1975), 509-517.

[7] Guy E. Blelloch, Jeremy T. Fineman, Phillip B. Gibbons, and Julian Shun. 2012. Internally Deterministic Parallel Algorithms Can Be Fast. In ACM SIGPLAN Symposium on Proceedings of Principles and Practice of Parallel Programming (PPoPP). 181-192.

[8] Guy E. Blelloch, Phillip B. Gibbons, and Harsha Vardhan Simhadri. 2010. Low-Depth Cache Oblivious Algorithms. In ACM Symposium on Parallelism in Algorithms and Architectures (SPAA). 189-199.

[9] Robert D. Blumofe and Charles E. Leiserson. 1999. Scheduling Multithreaded Computations by Work Stealing. f. ACM 46, 5 (Sept. 1999), 720-748.

[10] Christian Böhm, Robert Noll, Claudia Plant, and Bianca Wackersreuther. 2009. Density-based Clustering Using Graphics Processors. In ACM Conference on Information and Knowledge Management. 661-670.

[11] B. Borah and D. K. Bhattacharyya. 2004. An improved sampling-based DBSCAN for large spatial databases. In International Conference on Intelligent Sensing and Information Processing. 92-96.

[12] Prosenjit Bose, Anil Maheshwari, Pat Morin, Jason Morrison, Michiel Smid, and Jan Vahrenhold. 2007. Space-efficient geometric divideand-conquer algorithms. Computational Geometry 37, 3 (2007), 209 227.

[13] S. Brecheisen, H. Kriegel, and M. Pfeifle. 2004. Efficient density-based clustering of complex objects. In IEEE International Conference on Data Mining (ICDM). 43-50.

[14] Stefan Brecheisen, Hans-Peter Kriegel, and Martin Pfeifle. 2006. Parallel Density-Based Clustering of Complex Objects. In Advances in Knowledge Discovery and Data Mining (PAKDD). 179-188.

[15] Richard P. Brent. 1974. The Parallel Evaluation of General Arithmetic Expressions. 7. ACM 21, 2 (April 1974), 201-206.

[16] Ricardo Campello, Davoud Moulavi, Arthur Zimek, and Jörg Sander. 2015. Hierarchical Density Estimates for Data Clustering, Visualization, and Outlier Detection. ACM Trans. Knowl. Discov. Data 10, 1, Article 5 (July 2015), 5:1-5:51 pages.

[17] Chun-Chieh Chen and Ming-Syan Chen. 2015. HiClus: Highly Scalable Density-based Clustering with Heterogeneous Cloud. Procedia Computer Science 53 (2015), 149 - 157

[18] Danny Z. Chen, Michiel Smid, and Bin Xu. 2005. Geometric Algorithms for Density-Based Data Clustering. International Journal of Computational Geometry \& Applications 15, 03 (2005), 239-260.
[19] Danny Z Chen, Michiel Smid, and Bin Xu. 2005. Geometric algorithms for density-based data clustering. International fournal of Computational Geometry \& Applications 15, 03 (2005), 239-260.

[20] Xiaoming Chen, Wanquan Liu, Huining Qiu, and Jianhuang Lai. 2011. APSCAN: A parameter free algorithm for clustering. Pattern Recognition Letters 32, 7 (2011), 973 - 986.

[21] Richard Cole. 1988. Parallel Merge Sort. SIAM J. Comput. 17, 4 (Aug. 1988), 770-785.

[22] Richard Cole, Philip N. Klein, and Robert E. Tarjan. 1996. Finding Minimum Spanning Forests in Logarithmic Time and Linear Work Using Random Sampling. In ACM Symposium on Parallel Algorithms and Architectures (SPAA). 243-250.

[23] Massimo Coppola and Marco Vanneschi. 2002. High-performance Data Mining with Skeleton-based Structured Parallel Programming. Parallel Comput. 28, 5 (May 2002), 793-813.

[24] I. Cordova and T. Moh. 2015. DBSCAN on Resilient Distributed Datasets. In International Conference on High Performance Computing Simulation (HPCS). 531-540.

[25] Thomas H. Cormen, Charles E. Leiserson, Ronald L. Rivest, and Clifford Stein. 2009. Introduction to Algorithms (3. ed.). MIT Press.

[26] CriteoLabs. 2013. Terabyte Click Logs. http://labs.criteo.com/ downloads/download-terabyte-click-logs/

[27] B. Dai and I. Lin. 2012. Efficient Map/Reduce-Based DBSCAN Algorithm with Optimized Data Partition. In IEEE International Conference on Cloud Computing. 59-66.

[28] Mark de Berg, Otfried Cheong, Marc van Kreveld, and Mark Overmars. 2008. Computational Geometry: Algorithms and Applications. SpringerVerlag.

[29] Mark de Berg, Ade Gunawan, and Marcel Roeloffzen. 2017. Faster DB-scan and HDB-scan in Low-Dimensional Euclidean Spaces. In International Symposium on Algorithms and Computation (ISAAC). 25:125:13.

[30] Dheeru Dua and Casey Graff. 2017. UCI Machine Learning Repository. http://archive.ics.uci.edu/ml

[31] Y. El-Sonbaty, M. A. Ismail, and M. Farouk. 2004. An efficient density based clustering algorithm for large databases. In IEEE International Conference on Tools with Artificial Intelligence. 673-677.

[32] Martin Ester, Hans-Peter Kriegel, Jörg Sander, and Xiaowei Xu. 1996. A Density-based Algorithm for Discovering Clusters a Density-based Algorithm for Discovering Clusters in Large Spatial Databases with Noise. In International Conference on Knowledge Discovery and Data Mining (KDD). 226-231.

[33] Xiufen Fu, Yaguang Wang, Yanna Ge, Peiwen Chen, and Shaohua Teng. 2014. Research and Application of DBSCAN Algorithm Based on Hadoop Platform. In Pervasive Computing and the Networked World. 73-87.

[34] Junhao Gan and Yufei Tao. 2017. On the Hardness and Approximation of Euclidean DBSCAN. ACM Trans. Database Syst. 42, 3 (2017), 14:114:45.

[35] Hillel Gazit. 1991. An Optimal Randomized Parallel Algorithm for Finding Connected Components in a Graph. SIAM 7. Comput. 20, 6 (Dec. 1991), 1046-1067.

[36] J. Gil, Y. Matias, and U. Vishkin. 1991. Towards a theory of nearly constant time parallel algorithms. In IEEE Symposium on Foundations of Computer Science (FOCS). 698-710.

[37] Markus Götz, Christian Bodenstein, and Morris Riedel. 2015. HPDBSCAN: Highly Parallel DBSCAN. In Workshop on Machine Learning in High-Performance Computing Environments. Article 2, 2:1-2:10 pages.

[38] Yan Gu, Julian Shun, Yihan Sun, and Guy E. Blelloch. 2015. A TopDown Parallel Semisort. In ACM Symposium on Parallelism in Algorithms and Architectures (SPAA). 24-34. 
[39] Ade Gunawan. 2013. A faster algorithm for DBSCAN. Master's thesis, Eindhoven University of Technology.

[40] M. Haklay and P. Weber. 2008. OpenStreetMap: User-Generated Street Maps. IEEE Pervasive Computing 7, 4 (Oct 2008), 12-18.

[41] Shay Halperin and Uri Zwick. 1994. An Optimal Randomized Logarithmic Time Connectivity Algorithm for the EREW PRAM (Extended Abstract). In ACM Symposium on Parallel Algorithms and Architectures (SPAA). 1-10.

[42] Shay Halperin and Uri Zwick. 2001. Optimal Randomized EREW PRAM Algorithms for Finding Spanning Forests. fournal of Algorithms 39, 1 (2001), 1 - 46.

[43] D. Han, A. Agrawal, W. Liao, and A. Choudhary. 2016. A Novel Scalable DBSCAN Algorithm with Spark. In IEEE International Parallel and Distributed Processing Symposium Workshops (IPDPSW). 1393-1402.

[44] Qing He, Hai Xia Gu, Qin Wei, and Xu Wang. 2017. A Novel DBSCAN Based on Binary Local Sensitive Hashing and Binary-KNN Representation. Adv. in MM 2017 (2017), 3695323:1-3695323:9.

[45] Yaobin He, Haoyu Tan, Wuman Luo, Shengzhong Feng, and Jianping Fan. 2014. MR-DBSCAN: a scalable MapReduce-based DBSCAN algorithm for heavily skewed data. Frontiers of Computer Science 8, 1 (01 Feb 2014), 83-99.

[46] Xu Hu, Jun Huang, and Minghui Qiu. 2017. A Communication Efficient Parallel DBSCAN Algorithm Based on Parameter Server. In ACM on Conference on Information and Knowledge Management (CIKM). 21072110.

[47] Xiaojuan Hu, Lei Liu, Ningjia Qiu, Di Yang, and Meng Li. 2018. A MapReduce-based improvement algorithm for DBSCAN. Fournal of Algorithms \& Computational Technology 12, 1 (2018), 53-61.

[48] Fang Huang, Qiang Zhu, Ji Zhou, Jian Tao, Xiaocheng Zhou, Du Jin, Xicheng Tan, and Lizhe Wang. 2017. Research on the Parallelization of the DBSCAN Clustering Algorithm for Spatial Data Mining Based on the Spark Platform. Remote Sensing 9, 12 (2017).

[49] M. Huang and F. Bian. 2009. A Grid and Density Based Fast Spatial Clustering Algorithm. In International Conference on Artificial Intelligence and Computational Intelligence, Vol. 4. 260-263.

[50] J. Jaja. 1992. Introduction to Parallel Algorithms. Addison-Wesley Professional.

[51] Jennifer Jang and Heinrich Jiang. 2019. DBSCAN++: Towards fast and scalable density clustering. In International Conference on Machine Learning (ICML), Vol. 97. 3019-3029.

[52] Eshref Januzaj, Hans-Peter Kriegel, and Martin Pfeifle. 2004. DBDC: Density Based Distributed Clustering. In International Conference on Extending Database Technology (EDBT). 88-105.

[53] Eshref Januzaj, Hans-Peter Kriegel, and Martin Pfeifle. 2004. Scalable Density-based Distributed Clustering. In European Conference on Principles and Practice of Knowledge Discovery in Databases. 231-244.

[54] Hua Jiang, Jing Li, Shenghe Yi, Xiangyang Wang, and Xin Hu. 2011. A new hybrid method based on partitioning-based DBSCAN and ant clustering. Expert Systems with Applications 38, 8 (2011), 9373 - 9381.

[55] Karin Kailing, Hans-Peter Kriegel, and Peer Kröger. 2004. DensityConnected Subspace Clustering for High-Dimensional Data. In SIAM International Conference on Data Mining. 246-256.

[56] Jeong-Hun Kim, Jong-Hyeok Choi, Kwan-Hee Yoo, and Aziz Nasridinov. 2019. AA-DBSCAN: an approximate adaptive DBSCAN for finding clusters with varying densities. The fournal of Supercomputing 75, 1 (01 Jan 2019), 142-169.

[57] Younghoon Kim, Kyuseok Shim, Min-Soeng Kim, and June Sup Lee. 2014. DBCURE-MR: An efficient density-based clustering algorithm for large data using MapReduce. Information Systems 42 (2014), 15 35.

[58] Marzena Kryszkiewicz and Piotr Lasek. 2010. TI-DBSCAN: Clustering with DBSCAN by Means of theÂăTriangle Inequality. In Rough Sets and Current Trends in Computing. 60-69.

[59] YongChul Kwon, Dylan Nunley, Jeffrey P. Gardner, Magdalena Balazinska, Bill Howe, and Sarah Loebman. 2010. Scalable Clustering Algorithm for N-Body Simulations in a Shared-Nothing Cluster. In Scientific and Statistical Database Management. 132-150.

[60] Charles E. Leiserson. 2010. The Cilk++ concurrency platform. F. Supercomputing 51, 3 (2010).

[61] B. Liu. 2006. A Fast Density-Based Clustering Algorithm for Large Databases. In International Conference on Machine Learning and Cybernetics. 996-1000.

[62] Alessandro Lulli, Matteo Dell'Amico, Pietro Michiardi, and Laura Ricci. 2016. NG-DBSCAN: Scalable Density-based Clustering for Arbitrary Data. Proc. VLDB Endow. 10, 3 (Nov. 2016), 157-168.

[63] G. Luo, X. Luo, T. F. Gooch, L. Tian, and K. Qin. 2016. A Parallel DBSCAN Algorithm Based on Spark. In IEEE International Conferences on Big Data and Cloud Computing. 548-553.

[64] K. Mahesh Kumar and A. Rama Mohan Reddy. 2016. A Fast DBSCAN Clustering Algorithm by Accelerating Neighbor Searching Using Groups Method. Pattern Recogn. 58, C (Oct. 2016), 39-48.

[65] S. Mahran and K. Mahar. 2008. Using grid for accelerating densitybased clustering. In IEEE International Conference on Computer and Information Technology. 35-40.

[66] Md. Mostofa Ali Patwary, Suren Byna, Nadathur Rajagopalan Satish, Narayanan Sundaram, Zarija Lukić, Vadim Roytershteyn, Michael J. Anderson, Yushu Yao, Prabhat, and Pradeep Dubey. 2015. BD-CATS: Big Data Clustering at Trillion Particle Scale. In ACM/IEEE International Conference for High Performance Computing, Networking, Storage and Analysis (SC). Article 6, 6:1-6:12 pages.

[67] M. M. A. Patwary, D. Palsetia, A. Agrawal, W. k. Liao, F. Manne, and A. Choudhary. 2012. A new scalable parallel DBSCAN algorithm using the disjoint-set data structure. In ACM/IEEE International Conference on High Performance Computing, Networking, Storage and Analysis (SC). 62:1-62:11.

[68] M. M. A. Patwary, D. Palsetia, A. Agrawal, W. K. Liao, F. Manne, and A. Choudhary. 2013. Scalable parallel OPTICS data clustering using graph algorithmic techniques. In $A C M$ /IEEE International Conference for High Performance Computing, Networking, Storage and Analysis (SC). 49:1-49:12.

[69] Md. Mostofa Ali Patwary, Nadathur Satish, Narayanan Sundaram, Fredrik Manne, Salman Habib, and Pradeep Dubey. 2014. PARDICLE: Parallel Approximate Density-based Clustering. In ACM/IEEE International Conference for High Performance Computing, Networking, Storage and Analysis (SC). 560-571.

[70] Seth Pettie and Vijaya Ramachandran. 2002. A Randomized TimeWork Optimal Parallel Algorithm for Finding a Minimum Spanning Forest. SIAM f. Comput. 31, 6 (2002), 1879-1895.

[71] John H. Reif and Sandeep Sen. 1992. Optimal randomized parallel algorithms for computational geometry. Algorithmica 7, 1 (01 Jun 1992), 91-117.

[72] Jörg Sander, Martin Ester, Hans-Peter Kriegel, and Xiaowei Xu. 1998. Density-Based Clustering in Spatial Databases: The Algorithm GDBSCAN and Its Applications. Data Mining and Knowledge Discovery 2, 2 (01 Jun 1998), 169-194.

[73] A. Sarma, P. Goyal, S. Kumari, A. Wani, J. S. Challa, S. Islam, and N. Goyal. 2019. $\mu$ DBSCAN: An Exact Scalable DBSCAN Algorithm for Big Data Exploiting Spatial Locality. In IEEE International Conference on Cluster Computing (CLUSTER). 1-11.

[74] Erich Schubert, Jörg Sander, Martin Ester, Hans Peter Kriegel, and Xiaowei Xu. 2017. DBSCAN Revisited, Revisited: Why and How You Should (Still) Use DBSCAN. ACM Trans. Database Syst. 42, 3, Article 19 (July 2017), 19:1-19:21 pages. 
[75] J. Shun and G. E. Blelloch. 2014. Phase-Concurrent Hash Tables for Determinism. In ACM Symposium on Parallelism in Algorithms and Architectures (SPAA). 96-107.

[76] Julian Shun, Guy E. Blelloch, Jeremy T. Fineman, Phillip B. Gibbons, Aapo Kyrola, Harsha Vardhan Simhadri, and Kanat Tangwongsan. 2012. Brief announcement: the Problem Based Benchmark Suite. In ACM Symposium on Parallelism in Algorithms and Architectures (SPAA). 68-70.

[77] Hwanjun Song and Jae-Gil Lee. 2018. RP-DBSCAN: A Superfast Parallel DBSCAN Algorithm Based on Random Partitioning. In ACM International Conference on Management of Data (SIGMOD). 1173-1187.

[78] Cheng-Fa Tsai and Chien-Tsung Wu. 2009. GF-DBSCAN: A New Efficient and Effective Data Clustering Technique for Large Databases. In WSEAS International Conference on Multimedia Systems \& Signal Processing. 231-236.

[79] O. Uncu, W. A. Gruver, D. B. Kotak, D. Sabaz, Z. Alibhai, and C. Ng. 2006. GRIDBSCAN: GRId Density-Based Spatial Clustering of Applications with Noise. In IEEE International Conference on Systems, Man and Cybernetics, Vol. 4. 2976-2981.

[80] Uzi Vishkin. 2010. Thinking in Parallel: Some Basic Data-Parallel Algorithms and Techniques.

[81] P. Viswanath and V. Suresh Babu. 2009. Rough-DBSCAN: A fast hybrid density based clustering method for large data sets. Pattern Recognition Letters 30, 16 (2009), 1477 - 1488.

[82] P. Viswanath and R. Pinkesh. 2006. l-DBSCAN : A Fast Hybrid Density Based Clustering Method. In International Conference on Pattern
Recognition (ICPR), Vol. 1. 912-915.

[83] Yiqiu Wang, Yan Gu, and Julian Shun. 2019. Theoretically-Efficient and Practical Parallel DBSCAN. arXiv:cs.DS/1912.06255

[84] Benjamin Welton, Evan Samanas, and Barton P. Miller. 2013. Mr. Scan: Extreme Scale Density-based Clustering Using a Tree-based Network of GPGPU Nodes. In ACM/IEEE International Conference on High Performance Computing, Networking, Storage and Analysis (SC). Article 84, 84:1-84:11 pages.

[85] Yi-Pu Wu, Jin-Jiang Guo, and Xue-Jie Zhang. 2007. A Linear DBSCAN Algorithm Based on LSH. In International Conference on Machine Learning and Cybernetics, Vol. 5. 2608-2614.

[86] Yan Xiang Fu, Wei Zhong Zhao, and Huifang Ma. 2011. Research on parallel DBSCAN algorithm design based on MapReduce. Advanced Materials Research 301-303 (07 2011), 1133-1138.

[87] Xiaowei Xu, Jochen Jäger, and Hans-Peter Kriegel. 1999. A Fast Parallel Clustering Algorithm for Large Spatial Databases. Data Mining and Knowledge Discovery 3, 3 (01 Sep 1999), 263-290.

[88] Yanwei Yu, Jindong Zhao, Xiaodong Wang, Qin Wang, and Yonggang Zhang. 2015. Cludoop: An Efficient Distributed Density-based Clustering for Big Data Using Hadoop. Int. 7. Distrib. Sen. Netw. 2015, Article 2 (Jan. 2015), 2:2-2:2 pages.

[89] Yu Zheng, Like Liu, Longhao Wang, and Xing Xie. 2008. Learning Transportation Mode from Raw Gps Data for Geographic Applications on the Web. In International Conference on World Wide Web. 247-256. 